\title{
NORMAL FORMS FOR SEMILINEAR QUANTUM HARMONIC OSCILLATORS
}

\author{
by \\ Benoît Grébert, Rafik Imekraz, Eric Paturel
}

Abstract. - We consider the semilinear harmonic oscillator
\[ i \psi_{t}=\left(-\Delta+|x|^{2}+M\right) \psi+\partial_{2} g(\psi, \bar{\psi}), \quad x \in \mathbb{R}^{d}, t \in \mathbb{R} \]

where $M$ is a Hermite multiplier and $g$ a smooth function globally of order 3 at least.

We prove that such a Hamiltonian equation admits, in a neighborhood of the origin, a Birkhoff normal form at any order and that, under generic conditions on $M$ related to the non resonance of the linear part, this normal form is integrable when $d=1$ and gives rise to simple (in particular bounded) dynamics when $d \geq 2$.

As a consequence we prove the almost global existence for solutions of the above equation with small Cauchy data. Furthermore we control the high Sobolev norms of these solutions.

Résumé (Formes normales de Birkhoff pour l'oscillateur harmonique quantique non linéaire)

Dans cet article nous considérons l'oscillateur harmonique semi-linéaire :

$$
i \psi_{t}=\left(-\Delta+x^{2}+M\right) \psi+\partial_{2} g(\psi, \bar{\psi}), \quad x \in \mathbb{R}^{d}, t \in \mathbb{R}
$$

où $M$ est un multiplicateur de Hermite et $g$ est une fonction régulière globalement d'ordre au moins trois.

Nous montrons qu'une telle équation admet, au voisinage de zéro, une forme normale de Birkhoff à n'importe quel ordre et que, sous des hypothèses génériques sur $M$ liées à la non résonance de la partie linéaire, cette forme normale est complètement intégrable si $d=1$ et donne lieu à une dynamique simple (et en particulier bornée) pour $d \geq 2$.

Ce résultat nous permet de démontrer l'existence presque globale et de contrôler les normes de Sobolev d'indice grand des solutions de l'équation non linéaire ci-dessus avec donnée initiale petite.

Key words and phrases. - Birkhoff normal form, Semilinear quantum harmonic oscillator, Hamiltonian PDEs, long time stability, Gross-Pitaevskii equation. AMS classification: 37K55,37K45, 35B34, 35B35. 


\section{Introduction, statement of the results}

The aim of this paper is to prove a Birkhoff normal form theorem for the semilinear harmonic oscillator equation

$$
\left\{\begin{aligned}
i \psi_{t} & =\left(-\Delta+|x|^{2}+M\right) \psi+\partial_{2} g(\psi, \bar{\psi}) \\
\left.\psi\right|_{t=0} & =\psi_{0}
\end{aligned}\right.
$$

on the whole space $\mathbb{R}^{d}(d \geq 1)$ and to discuss its dynamical consequences. Here $g$ is a smooth function, globally of order $p \geq 3$ at 0 , and $\partial_{2} g$ denotes the partial derivative of $g$ with respect to the second variable. The linear operator $M$ is a Hermite multiplier. To define it precisely (at least in the case $d=1$, see Section 3.2 for the multidimensional case), let us introduce the quantum harmonic oscillator on $\mathbb{R}^{d}$, denoted by $T=-\Delta+|x|^{2}$. When $d=1, T$ is diagonal in the Hermite basis $\left(\phi_{j}\right)_{j \in \overline{\mathbb{N}}}$ :

$$
\begin{aligned}
T \phi_{j} & =(2 j-1) \phi_{j}, \quad j \in \overline{\mathbb{N}} \\
\phi_{n+1} & =\frac{H_{n}(x)}{\sqrt{2^{n} n !}} e^{-x^{2} / 2}, \quad n \in \mathbb{N}
\end{aligned}
$$

where $H_{n}(x)$ is the $n^{\text {th }}$ Hermite polynomial relative to the weight $e^{-x^{2}}$ :

$$
\int_{\mathbb{R}} e^{-x^{2}} H_{m}(x) H_{n}(x) d x=2^{n} n ! \sqrt{\pi} \delta_{n m} .
$$

In this basis (and for $d=1$ ), a Hermite multiplier is an operator given by

$$
M \phi_{j}=m_{j} \phi_{j}
$$

where $\left(m_{j}\right)_{j \in \overline{\mathbb{N}}}$ is a bounded sequence of real numbers, that will be chosen in the following classes : for any $k \geq 1$, we define the class

$$
\mathcal{W}_{k}=\left\{\left(m_{j}\right)_{j \in \overline{\mathbb{N}}} \mid \text { for each } j, m_{j}=\frac{\tilde{m}_{j}}{j^{k}} \text { with } \tilde{m}_{j} \in[-1 / 2,1 / 2]\right\}
$$

that we endow with the product probability measure. In this context the linear frequencies, i.e. the eigenvalues of $T+M=-d^{2} / d x^{2}+x^{2}+M$ are given by

$$
\omega_{j}=2 j-1+m_{j}=2 j-1+\frac{\tilde{m}_{j}}{j^{k}}, \quad j \in \overline{\mathbb{N}} .
$$

Let

$$
\begin{aligned}
& \tilde{H}^{s}=\left\{f \in H^{s}\left(\mathbb{R}^{d}, \mathbb{C}\right) \mid x \mapsto x^{\alpha} \partial^{\beta} f \in L^{2}\left(\mathbb{R}^{d}\right)\right. \\
& \left.\quad \text { for any } \alpha, \beta \in \mathbb{N}^{d} \text { satisfying } 0 \leq|\alpha|+|\beta| \leq s\right\}
\end{aligned}
$$

where $H^{s}\left(\mathbb{R}^{d}, \mathbb{C}\right)$ is the standard Sobolev space on $\mathbb{R}^{d}$. We note that, for any $s \geq 0$, the domain of $T^{s / 2}$ is $\tilde{H}^{s}$ (see for instance [Hel84] Proposition 1.6.6) 
and that for $s>d / 2, \tilde{H}^{s}$ is an algebra.

If $\psi_{0} \in \tilde{H}^{s}$ is small, say of norm $\epsilon$, local existence theory implies that (1.1) admits a unique solution in $\tilde{H}^{s}$ defined on an interval of length $c \epsilon^{-p+2}$. Our goal is to prove that for $M$ outside an exceptional subset, given any integer $r \geq 1$ and provided that $s$ is large enough and $\epsilon$ is small enough, the solution extends over an interval of length $c \epsilon^{-r}$. Furthermore we control the norm of the solution in $\tilde{H}^{s}$-norm $(d \geq 1)$ and localize the solution in the neighborhood of a torus (only in the case $d=1$, cf. Theorem 3.4 and Theorem 3.10).

Precisely we have

Theorem 1.1. - Let $r, k \in \mathbb{N}$ be arbitrary integers. There exists a set $F_{k} \subset$ $\mathcal{W}_{k}$ whose measure equals 1 such that if $m=\left(m_{j}\right)_{j \in \overline{\mathbb{N}}} \in F_{k}$ and if $g$ is a $C^{\infty}$ function on a neighborhood of the origin in $\mathbb{C}^{2}$, satisfying $g(z, \bar{z}) \in \mathbb{R}$ and vanishing at least at order 3 at the origin, there is $s_{0} \in \mathbb{N}$ such that for any $s \geq s_{0}$, $f$ there are $\epsilon_{0}>0, c>0$, such that for any $\epsilon \in\left(0, \epsilon_{0}\right)$, for any $\psi_{0}$ in $\tilde{H}^{s}$ with $\left\|\psi_{0}\right\|_{s} \leq \epsilon$, the Cauchy problem (1.1) with initial datum $\psi_{0}$ has a unique solution

$$
\psi \in C^{1}\left(\left(-T_{\epsilon}, T_{\epsilon}\right), \tilde{H}^{s}\right)
$$

with $T_{\epsilon} \geq c \epsilon^{-r}$. Moreover, for any $t \in\left(-T_{\epsilon}, T_{\epsilon}\right)$, one has

$$
\|\psi(t, \cdot)\|_{\tilde{H}^{s}} \leq 2 \epsilon .
$$

For the nonlinearity $g(\psi, \bar{\psi})=\lambda \frac{2}{p+1}|\psi|^{p+1}$ with $p \geq 1$ and without Hermite multiplier $(M=0)$, we recover the Gross-Pitaevskii equation

$$
i \psi_{t}=\left(-\Delta+|x|^{2}\right) \psi+\lambda|\psi|^{p-1} \psi, \quad t \in \mathbb{R}, \quad x \in \mathbb{R}^{d} .
$$

In this case, the global existence in the energy space $\tilde{H}^{1}$ has been proved for (1) $1 \leq p<\frac{d+2}{(d-2)^{+}}$without smallness assumption on the Cauchy data in the defocusing case $(\lambda<0)$ and for small Cauchy data in the focusing case $(\lambda>0)$ (see Car02 and also Zha05). But nothing is known for nonlinearities of higher order, neither about conservation of the $\tilde{H}^{s}$-norm for $s>1$. Our result states that, avoiding resonances by adding a generic linear term $M \psi$ (but $M=0$ is not allowed), we recover almost global existence for solutions of Gross-Pitaevskii equation with a nonlinearity of arbitrary high order and small Cauchy data in $\tilde{H}^{s}$ for $s$ large enough. In some sense, this shows that the instability for Gross-Pitaevskii that could appear in that regime are necessarily produced by resonances. More precisely, we can compare with

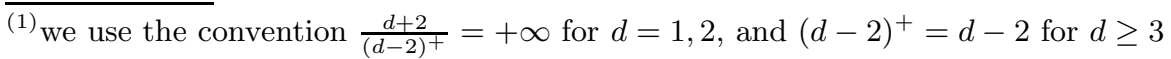


the semi-classical cubic Gross-Pitaevskii in $\mathbb{R}^{3}$ which appears in the study of Bose-Einstein condensates (for a physical presentation see [PS03])

$$
i h u_{t}=-h^{2} \Delta u+|x|^{2} u+h^{2}|u|^{2} u, \quad t \in \mathbb{R}, \quad x \in \mathbb{R}^{3}
$$

where $h$ is a small parameter.

The scaling relation between $\psi$ solution of (1.5) and $u$ solution of (1.6) is given by

$$
u(t, x)=\frac{1}{\sqrt{h}} \psi\left(t, \frac{x}{\sqrt{h}}\right) .
$$

We note that for multi indices $\alpha, \beta \in \mathbb{N}^{3}$, with $y=\frac{x}{\sqrt{h}}$,

$$
\left\|y^{\alpha} \partial^{\beta} \psi\right\|_{L^{2}\left(\mathbb{R}^{3}\right)}^{2}=h^{|\beta|-|\alpha|-1 / 2}\left\|x^{\alpha} \partial^{\beta} u\right\|_{L^{2}\left(\mathbb{R}^{3}\right)}^{2} .
$$

Thus the smallness of $\psi_{0}$ in $\tilde{H}^{s}$ imposed in Theorem 1.1, i.e. $\left\|\psi_{0}\right\|_{\tilde{H}^{s}} \leq C \epsilon$, reads

$$
\sum_{|\beta|+|\alpha| \leq s} h^{|\beta|-|\alpha|-1 / 2}\left\|x^{\alpha} \partial^{\beta} u_{0}\right\|_{L^{2}\left(\mathbb{R}^{3}\right)}^{2} \leq C \epsilon^{2} .
$$

Taking $\epsilon=h^{1 / 6}$ with $h$ small enough, this allows the derivatives of order greater than 1 to have large $L^{2}$-norm when $h$ is small:

$$
\left\|\partial^{\beta} u_{0}\right\|_{L^{2}\left(\mathbb{R}^{3}\right)}^{2}=O\left(h^{-|\beta|+5 / 6}\right)
$$

i.e. the initial data has to be small in $L^{2}$ but may have large oscillations. Then, Theorem 1.1 states that, avoiding the resonances by adding a generic linear term (which, in the preceding scaling, stays of order $h$ ), the same estimates remain true for the solution $u(t,$.$) with |t|=O\left(h^{-r / 6}\right), r$ being chosen arbitrarily from the principle. Notice that the role of the linear operator $M$ is to remove the resonances between the free modes (see (1.2)). The fully resonant case $M=0$ is beyond the scope of the paper.

To prove Theorem 1.1 we use the Birkhoff normal form theory. This technique has been developped by Bourgain Bou96, Bambusi Bam03, Bambusi-Grébert BG06] for semilinear PDEs (typically semilinear Schrödinger equation or semilinear wave equation) on the one dimensional torus and by Bambusi-Delort-Grébert-Szeftel [BDGS07] for the semilinear Klein-Gordon equation on the sphere $S^{d}$ (or a Zoll manifold). These cases were concerned with compact domains. In our work the domain is $\mathbb{R}^{d}$, the potential $x^{2}$ guarantees that the spectrum remains pure point, but the free modes of the harmonic oscillator are not so well localized. 
For general reference on Hamiltonian PDEs and their perturbations, see the recent monographies Cra00, Kuk00, Bou05, KP03]. We also note that in Kuk93, a KAM-like theorem is proved for (1.1) in one dimension and with special nonlinearities .

Let us describe roughly the general method. Consider a Hamiltonian system whose Hamiltonian function decomposes in a quadratic part, $H_{0}$ (associated to the linear part of the equation), and a perturbative nonlinear part $P$ (at least cubic): $H=H_{0}+P$. We assume that $H_{0}$ is diagonal in a Hilbert basis $\left(\phi_{j}\right)_{j \geq 1}$ of the phase space $\mathcal{P}: H_{0}=\sum_{j} \omega_{j} \xi_{j} \eta_{j}$ for $(\xi, \eta) \in \mathcal{P}$ and $\omega=\left(\omega_{j}\right)_{j \geq 1}$ is the vector of free frequencies (the eigenvalues of the linear part). In the harmonic oscillator case, the Hilbert basis is given by the Hermite functions and $\mathcal{P}=\ell^{2} \times \ell^{2}$. The heuristic idea could be resumed as follows: if the free modes do not interact linearly (i.e. if $\omega$ is non resonant), and if they do not interact too much via the nonlinear term, then the system will remain close to an integrable one, up to a nonlinear term of very high order, and thus the solutions will exist and stay under control during a very long time. More precisely, by a Birkhoff normal form approach we prove (cf. Theorem 2.23 which is our main theorem) that $H \sim H_{0}^{\prime}+P^{\prime}$ where $H_{0}^{\prime}$ is no more quadratic but remains integrable (in the case $d=1$ ) and $P^{\prime}$ is at least of order $r$, where $r$ can be chosen arbitrarily large as soon as we work in a sufficiently small neighborhood of the origin.

To guarantee the second condition, i.e. that the free modes do not interact too much via the nonlinear term, we have to control the integral of the product of three or more modes:

$$
a_{j}=\int_{D} \phi_{j_{1}}(x) \cdots \phi_{j_{k}}(x) d x
$$

where $D$ is the space domain $\left(\mathbb{R}^{d}\right.$ in our case) and $j$ is a multi-index in $\mathbb{N}^{k}, k$ being smaller than the fixed order $r$ and larger than 3. It turns out that, in our case, this control cannot be as good as in the cases of compact domains studied previously.

Let us consider ordered multi-indices $j$, i.e. such that $j_{1} \geq j_{2} \geq \cdots \geq j_{k}$. In [BDGS07, Gré07, Bam07] the following control was used: there exists $\nu>0$ and for any $N \geq 1$ there exists $C_{N}>0$ such that for all ordered $j$

$$
\left|a_{j}\right| \leq C_{N} j_{3}^{\nu}\left(\frac{j_{3}}{j_{3}+j_{1}-j_{2}}\right)^{N} \text {. }
$$

In the case of the harmonic oscillator, this estimate is false (cf [Wan08] where an equivalent is computed for four modes) and we are only able to prove the 
following: there exists $\nu>0$ and for any $N \geq 1$ there exists $C_{N}>0$ such that for all ordered $j$

$$
\left|a_{j}\right| \leq C_{N} \frac{j_{3}^{\nu}}{j_{1}^{1 / 24}}\left(\frac{\sqrt{j_{2} j_{3}}}{\sqrt{j_{2} j_{3}}+j_{1}-j_{2}}\right)^{N} .
$$

The difference could seem minimal but it is technically important:

$\sum_{j_{1}}\left(\frac{j_{3}}{j_{3}+j_{1}-j_{2}}\right)^{\mu} \sim C j_{3}$ for an uniform constant $C$ providing $\mu>1$ and similarly $\sum_{j_{1}}\left(\frac{\sqrt{j_{2} j_{3}}}{\sqrt{j_{2} j_{3}}+j_{1}-j_{2}}\right)^{\mu} \sim C \sqrt{j_{2} j_{3}}$ for $\mu>1$. In the first case, the extra term $j_{3}$ can be absorbed by changing the value of $\nu$ in (1.9) $\left(\nu^{\prime}=\nu+1\right)$. This is not possible in the second case. In some sense the perturbative nonlinearity is no longer short range (cf. Wan08).

Actually in the case studied in Bou96, Bam03, BG06, the linear modes (i.e. the eigenfunctions of the linear part) are localized around the exponentials $e^{i k x}$, i.e. the eigenfunctions of the Laplacian on the torus. In particular the product of eigenfunctions is close to an other eigenfunction which makes the control of (1.8) simpler. In the harmonic oscillator case, the eigenfunctions are not localized and the product of eigenfunctions has more complicated properties. Notice that, in the case of the semilinear Klein-Gordon equation on the sphere, the control of (1.8) is more complicated to obtain, but an estimate of type (1.9) is proved in [DS04 for the Klein-Gordon equation on Zoll manifolds.

From the point of view of a normal form, the substitution of (1.9) by (1.10) has the following consequence:

Consider a formal polynomial

$$
Q(\xi, \eta) \equiv Q(z)=\sum_{l=0}^{k} \sum_{j \in \mathbb{N}^{l}} a_{j} z_{j_{1}} \ldots z_{j_{l}}
$$

with coefficients $a_{j}$ satisfying (1.9). In Gré07] or Bam07, it is proved that its Hamiltonian vector field $X_{Q}$ is then regular from $\mathcal{P}_{s}=\ell_{s}^{2} \times \ell_{s}^{2}$ to $\mathcal{P}_{s}$ for all $s$ large enough (depending on $\nu$ ). In our present case, i.e. if $a_{j}$ only satisfy (1.10), which defines the class $\mathcal{T}^{\nu}$, then we prove that $X_{Q}$ is regular from $\mathcal{P}_{s}$ to $\mathcal{P}_{s^{\prime}}$ for all $s^{\prime}<s-1 / 2+1 / 24$ and $s$ large enough. This "loss of regularity" would of course complicate an iterative procedure, but it is bypassed in the following way: the nonlinearity $P$ is regular in the sense that $X_{P}$ maps $\mathcal{P}_{s}$ to $\mathcal{P}_{s}$ continuously for $s$ large enough (essentially because the space $\tilde{H}^{s}$ is an algebra for $s>d / 2$ ). On the other hand, we build at each step a

$\left.\left.\overline{{ }^{(2)} \text { here } l_{s}^{2}=\left\{\left(z_{l}\right)\right.}\left|\sum l^{2 s}\right| z_{l}\right|^{2}<\infty\right\}$ and corresponds to functions $\psi=\sum z_{l} \phi_{l}$ in $\tilde{H^{2 s}}$. 
canonical transform which preserves the regularity. Indeed, at each iteration, we compute the canonical transformation as the time 1 flow of a Hamiltonian $\chi$, and the solution of the so called homological equation gives rise to an extra term in (1.10) for the coefficient of the polynomial $\chi$ :

$$
\left|a_{j}\right| \leq C_{N} \frac{j_{3}^{\nu}}{j_{1}^{1 / 24}\left(1+j_{1}-j_{2}\right)}\left(\frac{\sqrt{j_{2} j_{3}}}{\sqrt{j_{2} j_{3}}+j_{1}-j_{2}}\right)^{N} .
$$

Using such an estimate on the coefficients (in the class (3) denoted $\mathcal{T}^{\nu,+}$ in Section 2.2), we prove in Proposition 2.13 that $X_{\chi}$ is regular from $\mathcal{P}_{s}$ to $\mathcal{P}_{s}$ for all $s$ large enough. Furthermore, we prove in Proposition 2.18 that the Poisson bracket of a polynomial in $\mathcal{T}^{\nu}$ with a polynomial in $\mathcal{T}^{\nu,+}$ is in $\mathcal{T}^{\nu^{\prime}}$ for some $\nu^{\prime}$ larger than $\nu$. So an iterative procedure is possible in $\mathcal{P}_{s}$.

This smoothing effect of the homological equations was already used by $\mathrm{S}$. Kuksin in Kuk87] (see also Kuk93, Pös96]). Notice that this is, in some sense, similar to the local smoothing property for Schrödinger equations with potentials superquadratic at infinity studied in [YZ04].

Our article is organized as follows: in Section 2 we state and prove a specific Birkhoff normal form theorem adapted to the loss of regularity that we explained above. In Section 3, we apply this theorem to the $1-d$ semilinear harmonic oscillator equation (Subsection 3.1) and we generalize it to cover the multidimensional case (Subsection 3.2).

Acknowledgements: it is a great pleasure to thank Dario Bambusi and Didier Robert for many helpful discussions. We thank both referees for useful suggestions.

\section{The Birkhoff normal form}

2.1. The abstract model. - To begin with, we give an abstract model of infinite dimensional Hamiltonian system. In Section 3 we will verify that the nonlinear harmonic oscillator can be described in this abstract framework. Throughout the paper, we denote $\overline{\mathbb{N}}=\mathbb{N} \backslash\{0\}$ and $\overline{\mathbb{Z}}=\mathbb{Z} \backslash\{0\}$. We work in the phase space $\mathcal{P}_{s} \equiv \mathcal{P}_{s}(\mathbb{C}):=\ell_{s}^{2}(\mathbb{C}) \times \ell_{s}^{2}(\mathbb{C})$ where, for $s \in \mathbb{R}_{+}, \ell_{s}^{2}(\mathbb{C}):=$ $\left\{\left.\left(a_{j}\right)_{j \geq 1} \in \mathbb{C}^{\overline{\mathbb{N}}}\left|\sum_{j \geq 1} j^{2 s}\right| a_{j}\right|^{2}<+\infty\right\}$ is a Hilbert space for the standard norm: $\|a\|_{s}^{2}=\sum_{j \geq 1}|j|^{2 s}\left|a_{j}\right|^{2}$. We denote $\mathcal{P}_{s}(\mathbb{R}):=\left\{(\xi, \bar{\xi}) \in \mathcal{P}_{s}(\mathbb{C})\right\}$ the "real" part of $\mathcal{P}_{s}(\mathbb{C})$. We shall denote a general point of $\mathcal{P}_{s}$ by $z=(\xi, \eta)$ with $z=\left(z_{j}\right)_{j \in \overline{\mathbb{Z}}}$,

\footnotetext{
${ }^{(3)}$ Actually in section 2.2 instead of $\mathcal{T}^{\nu}$ and $\mathcal{T}^{\nu,+}$, we consider more general classes $\mathcal{T}^{\nu, \beta}$ and $\mathcal{T}^{\nu, \beta,+}$ where the parameter $\beta$ plays the role of the exponent $1 / 24$ in (1.10) and (1.11)
} 
$\xi=\left(\xi_{j}\right)_{j \in \overline{\mathbb{N}}}, \eta=\left(\eta_{j}\right)_{j \in \overline{\mathbb{N}}}$ and the correspondence: $z_{j}=\xi_{j}, z_{-j}=\eta_{j}$ for all $j \in \overline{\mathbb{N}}$. Finally, for a Hamiltonian function $H$, the Hamiltonian vector field $X_{H}$ is defined by

$$
X_{H}(z)=\left(\left(-\frac{\partial H}{\partial \xi_{k}}\right)_{k \in \overline{\mathbb{N}}},\left(\frac{\partial H}{\partial \eta_{k}}\right)_{k \in \overline{\mathbb{N}}}\right) .
$$

Definition 2.1. - Let $s \geq 0$, we denote by $\mathcal{H}^{s}$ the space of Hamiltonian functions $H$ defined on a neighborhood $\mathcal{U}$ of the origin in $\mathcal{P}_{s} \equiv \mathcal{P}_{s}(\mathbb{C})$, satisfying $H(\xi, \bar{\xi}) \in \mathbb{R}$ (we say that $H$ is real) and

$$
H \in C^{\infty}(\mathcal{U}, \mathbb{C}) \quad \text { and } \quad X_{H} \in C^{\infty}\left(\mathcal{U}, \mathcal{P}_{s}\right),
$$

as well as every homogeneous polynomial $H_{k}$ appearing in the Taylor expansion of $H$ at 0 :

$$
H_{k} \in C^{\infty}(\mathcal{U}, \mathbb{C}) \quad \text { and } \quad X_{H_{k}} \in C^{\infty}\left(\mathcal{U}, \mathcal{P}_{s}\right)
$$

Remark 2.2. - This property, for Hamiltonians contributing to the nonlinearity, will in particular force them to be semilinear perturbations of the harmonic oscillator.

In particular the Hamiltonian vector fields of functions $F, G$ in $\mathcal{H}^{s}$ are in $\ell_{s}^{2}(\mathbb{C}) \times \ell_{s}^{2}(\mathbb{C})$ and we can define their Poisson bracket by

$$
\{F, G\}=i \sum_{j \geq 1} \frac{\partial F}{\partial \xi_{j}} \frac{\partial G}{\partial \eta_{j}}-\frac{\partial F}{\partial \eta_{j}} \frac{\partial G}{\partial \xi_{j}} .
$$

Notice that since for $P \in \mathcal{H}^{s}$, the vector field $X_{P}$ is a $C^{\infty}$ function from a neighborhood of $\mathcal{P}_{s}$ to $\mathcal{P}_{s}$ we have

Lemma 2.3. - Let $P \in \mathcal{H}^{s}$ such that $P$ vanishes up to order $r+1$ at the origin, that is :

$$
\forall k \leq r+1, \forall j \in \overline{\mathbb{Z}}^{k}, \frac{\partial^{k} P}{\partial z_{j_{1}} \ldots \partial z_{j_{k}}}(0)=0
$$

Then there exists $\varepsilon_{0}>0$ and $C>0$ such that, for $z \in \mathcal{P}_{s}$ satisfying $\|z\|_{s} \leq \varepsilon_{0}$, we have

$$
\left\|X_{P}(z)\right\|_{s} \leq C\|z\|_{s}^{r} .
$$

Our model of integrable system is the harmonic oscillator

$$
H_{0}=\sum_{j \geq 1} \omega_{j} \xi_{j} \eta_{j}
$$


where $\omega=\left(\omega_{j}\right)_{j \geq 1} \in \mathbb{R}^{\overline{\mathbb{N}}}$ is the frequency vector. We will assume that these frequencies grow at most polynomially, i.e. that there exist $C>0$ and $\bar{d} \geq 0$ such that for any $j \in \overline{\mathbb{N}}$,

$$
\left|\omega_{j}\right| \leq C|j|^{\bar{d}}
$$

in such a way that $H_{0}$ be well defined on $\mathcal{P}_{s}$ for $s$ large enough.

The perturbation term is a real function, $P \in \mathcal{H}^{s}$, having a zero of order at least 3 at the origin. Our Hamiltonian function is then given by

$$
H=H_{0}+P
$$

and Hamilton's canonical equations read

$$
\left\{\begin{array}{l}
\dot{\xi}_{j}=-i \omega_{j} \xi_{j}-i \frac{\partial P}{\partial \eta_{j}}, j \geq 1 \\
\dot{\eta}_{j}=i \omega_{j} \eta_{j}+i \frac{\partial P}{\partial \xi_{j}}, j \geq 1 .
\end{array}\right.
$$

Our theorem will require essentially two hypotheses: one on the perturbation $P$ (see Definition 2.6) and one on the frequency vector $\omega$ that we describe now.

For $j \in \overline{\mathbb{Z}}^{k}$ with $k \geq 3$, we define $\mu(j)$ as the third largest integer among $\left|j_{1}\right|, \ldots,\left|j_{k}\right|$. Then we set $S(j):=\left|j_{i_{1}}\right|-\left|j_{i_{2}}\right|$ where $\left|j_{i_{1}}\right|$ and $\left|j_{i_{2}}\right|$ are respectively the largest integer and the second largest integer among $\left|j_{1}\right|, \ldots,\left|j_{k}\right|$. In particular, if the multi-index $j$ is ordered i.e. if $\left|j_{1}\right| \geq \ldots \geq\left|j_{k}\right|$ then

$$
\mu(j):=\left|j_{3}\right| \text { and } S(j)=\left|j_{1}\right|-\left|j_{2}\right| .
$$

In Bam03, BG06, Gré07, Bam07, the non resonance condition on $\omega$ reads

Definition 2.4. - A frequency vector $\omega \in \mathbb{R}^{\overline{\mathbb{N}}}$ is non resonant if for any $r \in \overline{\mathbb{N}}$, there are $\gamma>0$ and $\delta>0$ such that for any $j \in \overline{\mathbb{N}}^{r}$ and any $1 \leq i \leq r$, one has

$$
\left|\omega_{j_{1}}+\cdots+\omega_{j_{i}}-\omega_{j_{i+1}}-\cdots-\omega_{j_{r}}\right| \geq \frac{\gamma}{\mu(j)^{\delta}}
$$

except in the case $\left\{j_{1}, \ldots, j_{i}\right\}=\left\{j_{i+1}, \ldots, j_{r}\right\}$.

In the harmonic oscillator cas 4 , we are able to work with a slightly refined non resonance condition

\footnotetext{
(4) The following holds, more generally, if the frequency vector is non resonant as in Definition 2.4 and satisfies the asymptotic: $\omega_{l} \sim l^{n}$ with $n \geq 1$.
} 
Definition 2.5. - A frequency vector $\omega \in \mathbb{R}^{\overline{\mathbb{N}}}$ is strongly non resonant if for any $r \in \overline{\mathbb{N}}$, there are $\gamma>0$ and $\delta>0$ such that for any $j \in \overline{\mathbb{N}}^{r}$ and any $1 \leq i \leq r$, one has

$$
\left|\omega_{j_{1}}+\cdots+\omega_{j_{i}}-\omega_{j_{i+1}}-\cdots-\omega_{j_{r}}\right| \geq \gamma \frac{1+S(j)}{\mu(j)^{\delta}}
$$

except if $\left\{j_{1}, \ldots, j_{i}\right\}=\left\{j_{i+1}, \ldots, j_{r}\right\}$.

This improvement of the non resonance condition is similar to the modification to the standard second Melnikov condition introduced first by S. Kuksin in [Kuk87] (see also Kuk93] and [Pös96]).

2.2. Polynomial structure. - For $j \in \overline{\mathbb{Z}}^{k}$ with $k \geq 3$, we have already defined $\mu(j)$ and $S(j)$, we now introduce

$$
B(j)=\left|j_{i_{2}} j_{i_{3}}\right|^{1 / 2}, \quad C(j)=\left|j_{i_{1}}\right|
$$

where $\left|j_{i_{1}}\right|,\left|j_{i_{2}}\right|$ and $\left|j_{i_{3}}\right|$ are respectively the first, the second and the third largest integer among $\left|j_{1}\right|, \ldots,\left|j_{k}\right|$. We also define

$$
A(j)=\frac{B(j)}{B(j)+S(j)} \text {. }
$$

In particular, if the multi-index $j$ is ordered i.e. if $\left|j_{1}\right| \geq \ldots \geq\left|j_{k}\right|$ then

$$
A(j)=\frac{\left|j_{2} j_{3}\right|^{1 / 2}}{\left|j_{2} j_{3}\right|^{1 / 2}+\left|j_{1}\right|-\left|j_{2}\right|}
$$

and

$$
C(j)=\left|j_{1}\right| .
$$

Definition 2.6. - Let $k \geq 3, \beta \in(0,+\infty)$ and $\nu \in[0,+\infty)$ and let

$$
Q(\xi, \eta) \equiv Q(z)=\sum_{j \in \overline{\mathbb{Z}}^{k}} a_{j} z_{j_{1}} \ldots z_{j_{l}}
$$

be a formal homogeneous polynomial of degree $k$ on $\mathcal{P}_{s}(\mathbb{C}) . Q$ is in the class $\mathcal{T}_{k}^{\nu, \beta}$ if for any $N \geq 1$ there exists a constant $c_{N}>0$ such that for all $j \in \overline{\mathbb{Z}}^{k}$

$$
\left|a_{j}\right| \leq c_{N} \frac{\mu(j)^{\nu}}{C(j)^{\beta}} A(j)^{N} .
$$

We will also use

Definition 2.7. - Let $k \geq 3, \beta \in[0,+\infty)$ and $\nu \in[0,+\infty)$ and let

$$
Q(\xi, \eta) \equiv Q(z)=\sum_{j \in \overline{\mathbb{Z}}^{k}} a_{j} z_{j_{1}} \ldots z_{j_{l}}
$$


be a formal homogeneous polynomial of degree $k$ on $\mathcal{P}_{s}(\mathbb{C}) . Q$ is in the class $\mathcal{T}_{k}^{\nu, \beta,+}$ if for any $N \geq 1$ there exists a constant $c_{N}>0$ such that for all $j \in \overline{\mathbb{Z}}^{k}$

$$
\left|a_{j}\right| \leq c_{N} \frac{\mu(j)^{\nu}}{C(j)^{\beta}(1+S(j))} A(j)^{N} .
$$

The best constants $c_{N}$ in (2.7) define a family of semi-norms for which $\mathcal{T}_{k}^{\nu, \beta}$ is a Fréchet space.

Remark 2.8. - Notice that the formula (2.6) does not give a unique representation of polynomials on $\mathcal{P}_{s}$. However, since the estimates (2.7) and (2.8) are symmetric with respect to the order of the indexes $j_{1}, \cdots, j_{k}$, this non uniqueness does not affect Definitions 2.6 and 2.7 .

Remark 2.9. - In the estimate (2.7), the numerator allows an increasing behaviour with respect to $\mu(j)$ that will be useful to control the small divisors. The denominator imposes a slightly decreasing behaviour with respect to the largest index $C(j)$ and a highly decreasing behaviour for monomials having their two modes of largest indexes that are not of the same order. This control is slightly better in $\mathcal{T}_{k}^{\nu, \beta,+}$.

Remark 2.10. - We will see in Proposition 2.13 that, if $\beta>1 / 2$ then $\mathcal{T}_{k}^{\nu, \beta} \subset \mathcal{H}^{s}$ for $s \geq \nu+1$. Unfortunately $\beta$ is not that large in the harmonic oscillator case, where the best we obtain is $\beta=1 / 24$. Thus $P \in \mathcal{T}_{k}^{\nu, \beta}$ does not imply $P \in \mathcal{H}^{s}$. Nevertheless, as we will see in Proposition 2.13, a polynomial in $\mathcal{T}_{k}^{\nu, \beta}$ is well defined and continuous on a neighborhood of the origin in $\mathcal{P}_{s}(\mathbb{C})$ for $s$ large enough. As a comparison, in Gré07, Bam07, our estimate (2.7) is replaced with

$$
\left|a_{j}\right| \leq C_{N} \frac{\mu(j)^{N+\nu}}{(\mu(j)+S(j))^{N}} .
$$

which is actually better than (2.7), since it implies the $\mathcal{H}^{s}$ regularity. This type of control on the coefficients $a_{j}$ was first introduced in [DS04] in the context of multilinear forms.

Definition 2.11. - Let $\nu \geq 0$ and $\beta \geq 0$. A function $P$ is in the class $\mathcal{T}^{\nu, \beta}$ if

- there exists $s_{0} \geq 0$ such that, for any $s \geq s_{0}$ there exists $\mathcal{U}_{s}$, a neighborhood of the origin in $\mathcal{P}_{s}$ such that $P \in C^{\infty}\left(\mathcal{U}_{s}, \mathbb{C}\right)$.

- $P$ has a zero of order at least 3 in 0.

- for each $k \geq 3$ the Taylor's expansion of degree $k$ of $P$ at zero belongs to $\otimes_{l=3}^{k} \mathcal{T}_{l}^{\nu, \beta}$. 
We now define the class of polynomials in normal form:

Definition 2.12. - Let $k=2 m$ be an even integer. A formal homogeneous polynomial $Z$ of degree $k$ on $\mathcal{P}_{s}$ is in normal form if it reads

$$
Z(z)=\sum_{j \in \overline{\mathbb{N}} m} b_{j} z_{j_{1}} z_{-j_{1}} \ldots z_{j_{m}} z_{-j_{m}}
$$

i.e. $Z$ depends only on the actions $I_{l}:=z_{l} z_{-l}=\xi_{l} \eta_{l}$.

The aim of the Birkhoff normal form theorem is to reduce a given Hamiltonian of the form $H_{0}+P$ with $P$ in $\mathcal{H}^{s}$ to a Hamiltonian of the form $Z+R$ where $Z$ is in normal form and $R$ remains very small, in the sense that it has a zero of high order at the origin.

We now review the properties of polynomials in the class $\mathcal{T}^{\nu, \beta}$.

Proposition 2.13. - Let $k \in \overline{\mathbb{N}}, \nu \in[0,+\infty), \beta \in[0,+\infty), s \in \mathbb{R}$ with $s>\nu+1$, and let $P \in \mathcal{T}_{k+1}^{\nu, \beta}$. Then

(i) $P$ extends as a continuous polynomial on $\mathcal{P}_{s}(\mathbb{C})$ and there exists a constant $C>0$ such that for all $z \in \mathcal{P}_{s}(\mathbb{C})$

$$
|P(z)| \leq C\|z\|_{s}^{k+1}
$$

(ii) For any $s^{\prime}<s+\beta-\frac{1}{2}$, the Hamiltonian vector field $X_{P}$ extends as a bounded function from $\mathcal{P}_{s}(\mathbb{C})$ to $\mathcal{P}_{s^{\prime}}(\mathbb{C})$. Furthermore, for any $s_{0} \in(\nu+$ $1, s]$, there is $C>0$ such that for any $z \in \mathcal{P}_{s}(\mathbb{C})$

$$
\left\|X_{P}(z)\right\|_{s^{\prime}} \leq C\|z\|_{s}\|z\|_{s_{0}}^{(k-1)} .
$$

(iii) Assume moreover that $P \in \mathcal{T}_{k+1}^{\nu, \beta,+}$ with $\beta>0$, then the Hamiltonian vector field $X_{P}$ extends as a bounded function from $\mathcal{P}_{s}(\mathbb{C})$ to $\mathcal{P}_{s}(\mathbb{C})$. Furthermore, for any $s_{0} \in(\nu+1, s]$, there is $C>0$ such that for any $z \in \mathcal{P}_{s}(\mathbb{C})$

$$
\left\|X_{P}(z)\right\|_{s} \leq C\|z\|_{s}\|z\|_{s 0}^{(k-1)} .
$$

(iv) Assume finally that $P \in \mathcal{T}_{k+1}^{\nu, \beta}$ and $P$ is in normal form in the sense of Definition 2.12, then the Hamiltonian vector field $X_{P}$ extends as a bounded function from $\mathcal{P}_{s}(\mathbb{C})$ to $\mathcal{P}_{s}(\mathbb{C})$. Furthermore, for any $s_{0} \in(\nu, s]$, there is $C>0$ such that for any $z \in \mathcal{P}_{s}(\mathbb{C})$

$$
\left\|X_{P}(z)\right\|_{s} \leq C\|z\|_{s}\|z\|_{s_{0}}^{(k-1)} .
$$

Remark 2.14. - Since homogeneous polynomials are their own Taylor expansion at 0 , assertions (iii) and (iv) imply that every element of $\mathcal{T}_{k+1}^{\nu, \beta,+}$, and every element of $\mathcal{T}_{k+1}^{\nu, \beta}$ in normal form is in $\mathcal{H}^{s}$. 
Proof. - (i) Let $P$ be an homogeneous polynomial of degree $k+1$ in $\mathcal{T}_{k+1}^{\nu, \beta}$ and for $z \in \mathcal{P}_{s}(\mathbb{C})$ write

$$
P(z)=\sum_{j \in \overline{\mathbb{Z}}^{k+1}} a_{j} z_{j_{1}} \ldots z_{j_{k+1}} .
$$

One has, using first (2.7) and then that $A(j) \leq 1, C(j) \geq 1$,

$$
\begin{aligned}
|P(z)| & \leq C \sum_{j \in \overline{\mathbb{Z}}^{k+1}} \frac{\mu(j)^{\nu}}{C(j)^{\beta}} A(j)^{N} \prod_{i=1}^{k+1}\left|z_{j_{i}}\right| \\
& \leq C \sum_{j \in \overline{\mathbb{Z}}^{k+1}} \frac{\mu(j)^{\nu}}{\prod_{i=1}^{k+1}\left|j_{i}\right|^{s}} \prod_{i=1}^{k+1}\left|j_{i}\right|^{s}\left|z_{j_{i}}\right| \\
& \leq C \sum_{j \in \overline{\mathbb{Z}}^{k+1}} \frac{1}{\prod_{i=1}^{k+1}\left|j_{i}\right|^{s-\nu}} \prod_{i=1}^{k+1}\left|j_{i}\right|^{s}\left|z_{j_{i}}\right| \\
& \leq C\left(\sum_{l \in \overline{\mathbb{Z}}} \frac{1}{|l|^{2 s-2 \nu}}\right)^{\frac{k+1}{2}}\|z\|_{s}^{k+1}
\end{aligned}
$$

where, in the last inequality, we used $k+1$ times the Cauchy-Schwarz inequality. Since $s>\nu+1 / 2$, the last sum converges and the first assertion is proved.

(ii) We have to estimate the derivative of polynomial $P$ with respect to any of its variables. Because of (2.7), given any $N$, we get

$$
\left|\frac{\partial P}{\partial z_{l}}\right| \leq C_{N}(k+1) \sum_{j \in \overline{\mathbb{Z}}^{k}} \frac{\mu(j, l)^{\nu}}{C(j, l)^{\beta}} A(j, l)^{N}\left|z_{j_{1}}\right| \ldots\left|z_{j_{k}}\right|
$$


where the quantities $\mu(j, l), C(j, l)$ and $A(j, l)$ are computed for the $k+1$-tuple made of $j_{1}, \ldots, j_{k}, l$. Furthermore

$$
\begin{aligned}
&\left\|X_{P}(z)\right\|_{s^{\prime}}^{2} \leq C \sum_{l \in \overline{\mathbb{Z}}}\left(\sum_{j \in \overline{\mathbb{Z}}^{k}} \frac{|l|^{s^{\prime}} \mu(j, l)^{\nu}}{C(j, l)^{\beta}} A(j, l)^{N}\left|z_{j_{1}}\right| \ldots\left|z_{j_{k}}\right|\right)^{2} \\
& \leq C(k !)^{2} \sum_{l \in \overline{\mathbb{Z}}}\left(\sum_{j \in \overline{\mathbb{Z}}_{>}^{k}} \frac{|l|^{s^{\prime}} \mu(j, l)^{\nu}}{C(j, l)^{\beta}} A(j, l)^{N}\left|z_{j_{1}}\right| \ldots\left|z_{j_{k}}\right|\right)^{2} \\
&(2.15) \quad \leq C^{\prime}|| z \mid \|_{s_{0}}^{2(k-3)} \sum_{l \in \overline{\mathbb{Z}}}\left(\sum_{\left|j_{1}\right| \geq\left|j_{2}\right| \geq\left|j_{3}\right|} \frac{|l|^{s^{\prime}} \mu(j, l)^{\nu}}{C(j, l)^{\beta}} A(j, l)^{N}\left|z_{j_{1}}\right|\left|z_{j_{2}}\right|\left|z_{j_{3}}\right|\right)^{2}
\end{aligned}
$$

where $\overline{\mathbb{Z}}_{>}^{k}$ denotes the set of ordered $k$-uples $\left(j_{1}, \ldots, j_{k}\right)$ such that $\left|j_{1}\right| \geq\left|j_{2}\right| \geq$ $\cdots \geq\left|j_{k}\right|$. We used the following result in the last inequality:

Lemma 2.15. - Given any $s \geq 0, s_{0}>\frac{1}{2}$ and $z \in \ell_{s+s_{0}}^{2}$ we have

$$
\sum_{j \in \overline{\mathbb{Z}}}|j|^{s}\left|z_{j}\right| \leq C_{s_{0}}|| z \|_{s+s_{0}}
$$

Proof. - This result is a simple consequence of Cauchy-Schwarz inequality :

$$
\sum_{j \in \overline{\mathbb{Z}}}|j|^{s}\left|z_{j}\right|=\sum_{j \in \overline{\mathbb{Z}}} \frac{1}{|j|^{s_{0}}}|j|^{s+s_{0}}\left|z_{j}\right| \leq\left(\sum_{j \in \overline{\mathbb{Z}}} \frac{1}{|j|^{2 s_{0}}}\right)^{\frac{1}{2}}\|z\|_{s+s_{0}} .
$$

Before continuing with the proof of assertion (ii) of Proposition 2.13, we give two technical lemmas which give an estimate of $A(j, l)$.

Lemma 2.16. - Given any ordered $k$-tuple $j \in \overline{\mathbb{Z}}_{>}^{k}$ and $l \in \overline{\mathbb{Z}}$, we have

$$
|l| A(j, l) \leq 2\left|j_{1}\right|
$$

Proof. - It is straightforward if $|l| \leq 2\left|j_{1}\right|$, since $A(j, l) \leq 1$. If not, the order is the following : $|l|>2\left|j_{1}\right|>\left|j_{1}\right| \geq\left|j_{2}\right|$ and

$$
|l| A(j, l)=\frac{|l| \sqrt{\left|j_{1} j_{2}\right|}}{\sqrt{\left|j_{1} j_{2}\right|}+|l|-\left|j_{1}\right|} \leq \frac{|l| \sqrt{\left|j_{1} j_{2}\right|}}{|l| / 2} \leq 2\left|j_{1}\right|,
$$

and the lemma is proved. 
Lemma 2.17. - Given any ordered $k$-uple $j \in \overline{\mathbb{Z}}_{>}^{k}$ and $l \in \overline{\mathbb{Z}}$ we have

$$
A(j, l) \leq \tilde{A}\left(j_{1}, j_{2}, l\right):=\left\{\begin{array}{cl}
2 \frac{\left|j_{2}\right|}{|l|+\left|j_{1}\right|-\left|j_{2}\right|} & \text { if }|l| \leq\left|j_{2}\right| \\
2 \frac{\sqrt{\left|l j_{2}\right|}}{\sqrt{\left|l j_{2}\right|}+|| j_{1}|-| l||} & \text { if }|l| \geq\left|j_{2}\right| .
\end{array}\right.
$$

Proof. - If $|l|>2\left|j_{1}\right|, A(j, l)$ reads :

$$
A(j, l)=\frac{\sqrt{\left|j_{1} j_{2}\right|}}{\sqrt{\left|j_{1} j_{2}\right|}+|l|-\left|j_{1}\right|} .
$$

We can write :

$$
\begin{aligned}
\sqrt{\left|j_{1} j_{2}\right|}+|l|-\left|j_{1}\right| & =\sqrt{\left|l j_{2}\right|}+|l|-\left|j_{1}\right|-\sqrt{\left|j_{2}\right|}\left(\sqrt{|l|}-\sqrt{\left|j_{1}\right|}\right) \\
& =\sqrt{\left|l j_{2}\right|}+|l|-\left|j_{1}\right|-\sqrt{\left|j_{2}\right|} \frac{|l|-\left|j_{1}\right|}{\sqrt{|l|}+\sqrt{\left|j_{1}\right|}} \\
& \geq \sqrt{\left|l j_{2}\right|}+|l|-\left|j_{1}\right|-\sqrt{\left|j_{1}\right|} \frac{|l|-\left|j_{1}\right|}{\sqrt{|l|}+\sqrt{\left|j_{1}\right|}} \\
& \geq \sqrt{\left|l j_{2}\right|}+\frac{\sqrt{2}}{\sqrt{2}+1}\left(|l|-\left|j_{1}\right|\right)
\end{aligned}
$$

Hence,

$$
\begin{gathered}
A(j, l) \leq \frac{1+\sqrt{2}}{\sqrt{2}} \frac{\sqrt{\left|j_{1} j_{2}\right|}}{\sqrt{\left|j_{1} j_{2}\right|}+|l|-\left|j_{1}\right|} \leq 2 \frac{\sqrt{\left|l j_{2}\right|}}{\sqrt{\left|l j_{2}\right|}+|l|-\left|j_{1}\right|} . \\
\text { If }\left|j_{2}\right| \leq|l| \leq 2\left|j_{1}\right|, \text { then } B(j, l)^{2}=\left|j_{2}\right| \min \left(|l|,\left|j_{1}\right|\right) \in\left[\frac{\left|l j_{2}\right|}{2},\left|l j_{2}\right|\right], \text { therefore } \\
A(j, l) \leq \frac{\sqrt{\left|l j_{2}\right|}}{1 / \sqrt{2} \sqrt{\left|l j_{2}\right|}+|| l|-| j_{1}||} \leq 2 \frac{\sqrt{\left|l j_{2}\right|}}{\sqrt{\left|l j_{2}\right|}+|| l|-| j_{1}||} .
\end{gathered}
$$

Finally, if $|l| \leq\left|j_{2}\right|$ we get

$$
A(j, l)=\frac{\sqrt{\left|l j_{2}\right|}}{\sqrt{\left|l j_{2}\right|}+\left|j_{1}\right|-\left|j_{2}\right|} \leq 2 \frac{\left|j_{2}\right|}{|l|+\left|j_{1}\right|-\left|j_{2}\right|},
$$

and this ends the proof of Lemma 2.17

To continue with the proof of assertion (ii) of Proposition 2.13, we define $0<\varepsilon<s-s^{\prime}-\frac{1}{2}$, and $N=s+1+\varepsilon$. In view of (2.15), we may decompose :

$$
\left\|X_{P}(z)\right\|_{s^{\prime}}^{2} \leq C \sum_{l \in \overline{\mathbb{Z}}}\left(T_{1}(l)+T_{2}(l)\right)^{2},
$$


with

$$
\begin{aligned}
& T_{1}(l)=\sum_{\left|j_{1}\right| \geq\left|j_{2}\right| \geq\left|j_{3}\right|,\left|j_{2}\right|>|l|} \frac{|l|^{s^{\prime}}|\mu(j, l)|^{\nu}}{\max \left(\left|j_{1}\right|,|l|\right)^{\beta}} A(j, l)^{N}\left|z_{j_{1}}\right|\left|z_{j_{2}}\right|\left|z_{j_{3}}\right| \\
& T_{2}(l)=\sum_{\left|j_{1}\right| \geq\left|j_{2}\right| \geq\left|j_{3}\right|,\left|j_{2}\right| \leq|l|} \frac{|l|^{s^{\prime}}|\mu(j, l)|^{\nu}}{\max \left(\left|j_{1}\right|,|l|\right)^{\beta}} A(j, l)^{N}\left|z_{j_{1}}\right|\left|z_{j_{2}}\right|\left|z_{j_{3}}\right| .
\end{aligned}
$$

Since $A(j, l) \leq 1$ and $N>\frac{1}{2}+s^{\prime}+\varepsilon$, we may estimate $T_{1}(l)$ using Lemmas 2.16 and 2.17:

$$
\begin{aligned}
T_{1}(l) & \leq C \sum_{\left|j_{1}\right| \geq\left|j_{2}\right| \geq\left|j_{3}\right|,\left|j_{2}\right|>|l|}\left|j_{1}\right|^{s^{\prime}}\left|j_{2}\right|^{\nu} \tilde{A}\left(j_{1}, j_{2}, l\right)^{\frac{1}{2}+\varepsilon}\left|z_{j_{1}}\right|\left|z_{j_{2}}\right|\left|z_{j_{3}}\right| \\
& \leq C|| z||_{s_{0}} \sum_{\left|j_{1}\right| \geq\left|j_{2}\right|,\left|j_{2}\right|>|l|} \frac{1}{|l|^{\frac{1}{2}+\varepsilon}}\left|j_{1}\right|^{s-\frac{1}{2}-\varepsilon}\left|z_{j_{1}}\right|\left|j_{2}\right|^{\nu+\frac{1}{2}+\varepsilon}\left|z_{j_{2}}\right| \\
& \leq C|| z||_{s_{0}} \frac{1}{|l|^{\frac{1}{2}+\varepsilon}}|| z|| s|| z||_{\nu+1+2 \varepsilon}
\end{aligned}
$$

hence $T_{1}(l)$ is an $\ell^{2}$-sequence, whose $\ell^{2}$-norm is bounded above by $C\|z\|_{s_{0}}^{2}\|z\|_{s}$ if we assume that $s_{0}>\nu+1+2 \varepsilon$. Concerning $T_{2}(l)$, using Lemmas 2.16 and 2.17, we obtain

$$
\begin{aligned}
T_{2}(l) & \leq C \sum_{\left|j_{1}\right| \geq\left|j_{2}\right| \geq\left|j_{3}\right|,\left|j_{2}\right| \leq|l|} \frac{1}{|l|^{s-s^{\prime}+\beta}}\left|j_{1}\right|^{s}\left|j_{2}\right|^{\nu} \tilde{A}\left(j_{1}, j_{2}, l\right)^{N-s}\left|z_{j_{1}}\right|\left|z_{j_{2}}\right|\left|z_{j_{3}}\right| \\
& \leq C \frac{\| z||_{s_{0}}}{|l|^{s-s^{\prime}+\beta}} \sum_{\left|j_{1}\right| \geq\left|j_{2}\right|,\left|j_{2}\right| \leq|l|}\left(\frac{\sqrt{\left|l j_{2}\right|}}{1+|| j_{1}|-| l||}\right)^{1+\varepsilon}\left|j_{1}\right|^{s}\left|z_{j_{1}}\right|\left|j_{2}\right|^{\nu}\left|z_{j_{2}}\right| \\
& \leq C \frac{|| z||_{s_{0}}}{|l|^{s-s^{\prime}+\beta-(1+\varepsilon) / 2}}\left(\sum_{j_{2} \in \overline{\mathbb{Z}}}\left|j_{2}\right|^{\nu+(1+\varepsilon) / 2}\left|z_{j_{2}}\right|\right) \sum_{j_{1} \in \overline{\mathbb{Z}}} \frac{\left|j_{1}\right|^{s}\left|z_{j_{1}}\right|}{\left(1+|| j_{1}|-| l||\right)^{1+\varepsilon}}
\end{aligned}
$$

The last sum in $j_{1}$ is a convolution product of the $\ell^{2}$-sequence $\left|j_{1}\right|^{s}\left|z_{j_{1}}\right|$ and the $\ell^{1}$-sequence $\frac{1}{\left(1+\left|j_{1}\right|\right)^{1+\varepsilon}}$ and thus a $\ell^{2}$-sequence with respect to the index $l$, whose $\ell^{2}$-norm is bounded by $\|z\|_{s}$. Choosing $\varepsilon>0$ in such a way that $s-s^{\prime}+\beta-(1+\varepsilon) / 2>0$, the sequence $T_{2}(l)$ is in $\ell^{2}$, with a norm bounded by

$$
\left\|T_{2}\right\| \leq C\|z\|_{s_{0}}\|z\|_{\nu+(1+\varepsilon) / 2}\|z\|_{s} \leq C\|z\|_{s_{0}}^{2}\|z\|_{s},
$$

with $s_{0}>\nu+(1+\varepsilon) / 2$. Collecting the estimates for $T_{1}$ and $T_{2}$, we obtain the desired inequality. 
(iii) We define $0<\varepsilon<1 / 12$ and $N=s+\frac{1}{2}+\varepsilon$. We have, as in (ii), this first estimate

$$
\left\|X_{P}(z)\right\|_{s}^{2} \leq C\|z\|_{s_{0}}^{2(k-3)} \sum_{l \in \overline{\mathbb{Z}}}\left(\sum_{\left|j_{1}\right| \geq\left|j_{2}\right| \geq\left|j_{3}\right|} \frac{|l|^{s} \mu(j, l)^{\nu}}{C(j, l)^{\beta}(1+S(j, l))} A(j, l)^{N}\left|z_{j_{1}}\right|\left|z_{j_{2}}\right|\left|z_{j_{3}}\right|\right)^{2} .
$$

As in (ii), we may also decompose the sum on $j_{1}, j_{2}$ and $j_{3}$ into two pieces, $T_{1}^{+}(l)$ collecting all the terms with $\left|j_{2}\right|>|l|$ and $T_{2}^{+}(l)$ collecting those with $\left|j_{2}\right| \leq|l|$. Following (ii), since $C(j, l) \geq 1$ and $1+S(j, l) \geq 1$, we obtain for $T_{1}^{+}$:

$$
\begin{aligned}
T_{1}^{+}(l) & \leq C \sum_{\left|j_{1}\right| \geq\left|j_{2}\right| \geq\left|j_{3}\right|,\left|j_{2}\right|>|l|}|l|^{s}\left|j_{2}\right|^{\nu} A(j, l)^{N}\left|z_{j_{1}}\right|\left|z_{j_{2}}\right|\left|z_{j_{3}}\right| \\
& \leq C \sum_{\left|j_{1}\right| \geq\left|j_{2}\right| \geq\left|j_{3}\right|,\left|j_{2}\right|>|l|}|l|^{1 / 2+\varepsilon}\left|j_{1}\right|^{s-1 / 2-\varepsilon}\left|j_{2}\right|^{\nu} A(j, l)^{N-(s-1 / 2-\varepsilon)}\left|z_{j_{1}}\right|\left|z_{j_{2}}\right|\left|z_{j_{3}}\right| \\
& \leq\left. C|| z\right|_{s_{0}} \sum_{\left|j_{1}\right| \geq\left|j_{2}\right|,\left|j_{2}\right|>|l|}|l|^{1 / 2+\varepsilon}\left|j_{1}\right|^{s-1 / 2-\varepsilon}\left|j_{2}\right|^{\nu} \tilde{A}\left(j_{1}, j_{2}, l\right)^{N-(s-1 / 2-\varepsilon)}\left|z_{j_{1}}\right|\left|z_{j_{2}}\right| \\
& \leq C \|\left. z\right|_{s_{0}} \sum_{\left|j_{1}\right| \geq\left|j_{2}\right|,\left|j_{2}\right|>|l|}|l|^{s-N}\left|j_{1}\right|^{s-1 / 2-\varepsilon}\left|j_{2}\right|^{\nu+N-(s-1 / 2-\varepsilon)}\left|z_{j_{1}}\right|\left|z_{j_{2}}\right| \\
& \leq C|| z||_{s_{0}} \frac{1}{|l|^{\frac{1}{2}+\varepsilon}} \sum_{\left|j_{1}\right| \geq\left|j_{2}\right|,\left|j_{2}\right|>|l|}\left|j_{1}\right|^{s-1 / 2-\varepsilon}\left|j_{2}\right|^{\nu+N-(s-1 / 2-\varepsilon)}\left|z_{j_{1}}\right|\left|z_{j_{2}}\right| \\
& \leq C\|z\|_{s_{0}} \frac{1}{|l|^{\frac{1}{2}+\varepsilon}}|| z||_{s}|| z||_{\nu+1+2 \varepsilon},
\end{aligned}
$$

hence $T_{1}^{+}(l)$ is a $\ell^{2}$-sequence, whose $\ell^{2}$-norm is bounded above by $C\|z\|_{s_{0}}^{2}\|z\|_{s}$ if $s_{0}>\nu+1+2 \varepsilon$.

The estimate on $T_{2}^{+}$will need all factors assigned in the definition of $\mathcal{T}^{\nu, \beta,+}$ :

$$
\begin{aligned}
T_{2}^{+}(l) & \leq C \sum_{\left|j_{1}\right| \geq\left|j_{2}\right| \geq\left|j_{3}\right|,\left|j_{2}\right| \leq|l|} \frac{\left|j_{1}\right|^{s}\left|j_{2}\right|^{\nu}}{\max \left(j_{1}, l\right)^{\beta}\left(1+|| j_{1}|-| l||\right)} \tilde{A}\left(j_{1}, j_{2}, l\right)^{N-s}\left|z_{j_{1}}\right|\left|z_{j_{2}}\right|\left|z_{j_{3}}\right| \\
& \leq\left. C\|z\|\right|_{s_{0}} \sum_{\left|j_{1}\right| \geq\left|j_{2}\right|,\left|j_{2}\right| \leq|l|}\left(\frac{\sqrt{\left|l j_{2}\right|}}{1+|| j_{1}|-| l||}\right)^{\varepsilon} \frac{1}{|l|^{\beta}\left(1+|| j_{1}|-| l||\right)}\left|j_{1}\right|^{s}\left|z_{j_{1}}\right|\left|j_{2}\right|^{\nu}\left|z_{j_{2}}\right| \\
& \leq C \| z||_{s_{0}} \frac{1}{|l|^{\beta-\varepsilon / 2}} \sum_{j_{2} \in \overline{\mathbb{Z}}}\left|j_{2}\right|^{\nu+\varepsilon / 2}\left|z_{j_{2}}\right| \sum_{j_{1} \in \overline{\mathbb{Z}}} \frac{\left|j_{1}\right|^{s}\left|z_{j_{1}}\right|}{\left(1+|| j_{1}|-| l||\right)^{1+\varepsilon}} .
\end{aligned}
$$

Once again, the last sum in $j_{1}$ is a convolution product of the $\ell^{2}$ sequence $\left|j_{1}\right|^{s}\left|z_{j_{1}}\right|$ and the $\ell^{1}$ sequence $\frac{1}{\left(1+\left|j_{1}\right|\right)^{1+\varepsilon}}$. Choosing $\varepsilon>0$ in such a way that 
$\beta-\varepsilon / 2>0$, the sequence $T_{2}^{+}(l)$ is in $\ell^{2}$, with a norm bounded by

$$
\left\|T_{2}\right\| \leq C\|z\|_{s_{0}}\|z\|_{\nu+(1+\varepsilon) / 2}\|z\|_{s} \leq C\|z\|_{s_{0}}^{2}\|z\|_{s},
$$

with $s_{0}>\nu+(1+\varepsilon) / 2$. Collecting the estimates for $T_{1}^{+}$and $T_{2}^{+}$, we obtain the stated inequality.

(iv) Let $k+1=2 m$. As in (ii), we obtain

$$
\left\|X_{P}\right\|_{s}^{2} \leq C \sum_{l \in \overline{\mathbb{Z}}}\left(\sum_{j \in \overline{\mathbb{N}}_{>}^{m-1}}|l|^{s}\left|z_{l}\right| \frac{\mu(j, j, l, l)^{\nu}}{C(j, j, l, l)^{\beta}}\left|z_{j_{1}}\right|\left|z_{-j_{1}}\right| \ldots\left|z_{j_{m-1}}\right|\left|z_{-j_{m-1}}\right|\right)^{2},
$$

using the same convention for $\mu(j, j, l, l)$ and $C(j, j, l, l)$ as for $\mu(j, l)$ and $C(j, l)$ : as an example, $\mu(j, j, l, l)$ is the third biggest integer among $\left|j_{1}\right|,\left|j_{1}\right|, \ldots\left|j_{m-1}\right|,\left|j_{m-1}\right|,|l|$ and $|l|$, that is, if $j$ is ordered, either $\mu(j, j, l, l)=$ $\left|j_{1}\right|$, and in this case $C(j, j, l, l)=|l|$, or $\mu(j, j, l, l)=|l|$ and in this case $C(j, j, l, l)=\left|j_{1}\right|$. Notice that $A(j, j, l, l)=1$ does not help for this computation. The sum over $j$ can be decomposed into two parts :

$$
\begin{aligned}
& \sum_{j \in \overline{\mathbb{N}}_{>}^{m-1}, j_{1} \leq l}|l|^{s}\left|z_{l}\right| \frac{\mu(j, j, l, l)^{\nu}}{C(j, j, l, l)^{\beta}}\left|z_{j_{1}}\right|\left|z_{-j_{1}}\right| \ldots\left|z_{j_{m-1}}\right|\left|z_{-j_{m-1}}\right| \\
\leq & \sum_{j \in \overline{\mathbb{N}}_{>}^{m-1}, j_{1} \leq l}|l|^{s}\left|z_{l}\right| \frac{\left|j_{1}\right|^{\nu}}{|l|^{\beta}}\left|z_{j_{1}}\right|\left|z_{-j_{1}}\right| \ldots\left|z_{j_{m-1}}\right|\left|z_{-j_{m-1}}\right| \\
\leq & |l|^{s-\beta}\left|z_{l}\right| \sum_{j_{1}} j_{1}^{\nu}\left|z_{j_{1}}\right|\left|z_{-j_{1}}\right||| z \|_{0}^{2(m-2)} \\
\leq & |l|^{s-\beta}\left|z_{l}\right||| z\left\|_{\nu / 2}^{2}|| z\right\|_{0}^{2(m-2)}
\end{aligned}
$$

and

$$
\begin{aligned}
& \sum_{j \in \overline{\mathbb{N}}_{>}^{m-1}, j_{1}>l}|l|^{s}\left|z_{l}\right| \frac{\mu(j, j, l, l)^{\nu}}{C(j, j, l, l)^{\beta}}\left|z_{j_{1}}\right|\left|z_{-j_{1}}\right| \ldots\left|z_{j_{m-1}}\right|\left|z_{-j_{m-1}}\right| \\
\leq & |l|^{s}\left|z_{l}\right| \sum_{j \in \overline{\mathbb{N}}_{>}^{m-1}}\left|j_{1}\right|^{\nu-\beta}\left|z_{j_{1}}\right|\left|z_{-j_{1}}\right| \ldots\left|z_{j_{m-1}}\right|\left|z_{-j_{m-1}}\right| \\
\leq & |l|^{s}\left|z_{l}\right||| z\left\|_{(\nu-\beta) / 2}^{2}|| z\right\|_{0}^{2(m-2)}
\end{aligned}
$$

Inserting these two estimates in (2.18) we get (2.12).

The second essential property satisfied by polynomials in $\mathcal{T}_{k}^{\nu, \beta}$ is captured in the following 
Proposition 2.18. - Let $k_{1}, k_{2} \geq 2, \nu_{1}, \nu_{2} \geq 0$ and $\beta>0$ The map $(P, Q) \mapsto\{P, Q\}$ defines a continuous map from $\mathcal{T}_{k_{1}+1}^{\nu_{1}, \beta,+} \times \mathcal{T}_{k_{2}+1}^{\nu_{2}, \beta}$ to $\mathcal{T}_{k_{1}+k_{2}}^{\nu^{\prime}, \beta}$ for $\nu^{\prime}=2\left(\nu_{1}+\nu_{2}\right)+1$.

Proof. - We assume that $P \in \mathcal{T}_{k_{1}+1}^{\nu_{1}, \beta,+}$ and $Q \in \mathcal{T}_{k_{2}+1}^{\nu_{2}, \beta}$ are homogeneous polynomials and we write

$$
P(z)=\sum_{j \in \overline{\mathbb{Z}}^{k_{1}+1}} a_{j} z_{j_{1}} \ldots z_{j_{k_{1}+1}}
$$

and

$$
Q(z)=\sum_{i \in \overline{\mathbb{Z}}^{k_{2}+1}} b_{i} z_{i_{1}} \ldots z_{i_{k_{2}+1}} .
$$

In view of the symmetry of the estimate (2.7) with respect to the involved indices, one easily obtains

$$
\{P, Q\}(z)=\sum_{(j, i) \in \overline{\mathbb{Z}}^{k_{1}+k_{2}}} c_{j, i} z_{j_{1}} \ldots z_{j_{k_{1}}} z_{i_{1}} \ldots z_{i_{k_{2}}}
$$

with

$$
\left|c_{j, i}\right| \leq c_{N, N^{\prime}} \sum_{l \in \overline{\mathbb{Z}}} \frac{\mu(j, l)^{\nu_{1}}}{C(j, l)^{\beta}(1+S(j, l))} A(j, l)^{N} \frac{\mu(i, l)^{\nu_{2}}}{C(i, l)^{\beta}} A(i, l)^{N^{\prime}} .
$$

Therefore it remains to prove that, for each $M \geq 1$, there exist $N, N^{\prime} \geq 1$, $C>0$ such that for all $j \in \overline{\mathbb{Z}}^{k_{1}}$ and all $i \in \overline{\mathbb{Z}}^{k_{2}}$,

$$
\sum_{l \in \overline{\mathbb{Z}}} \frac{\mu(j, l)^{\nu_{1}}}{C(j, l)^{\beta}(1+S(j, l))} A(j, l)^{N} \frac{\mu(i, l)^{\nu_{2}}}{C(i, l)^{\beta}} A(i, l)^{N^{\prime}} \leq C \frac{\mu(j, i)^{\nu^{\prime}}}{C(i, j)^{\beta}} A(j, i)^{M}
$$

with $\nu^{\prime}=2\left(\nu_{1}+\nu_{2}\right)+1$.

In order to simplify the notations, and because it does not change the estimates of (2.19), we will assume that $k_{1}=k_{2}=k$. We can also assume by symmetry that

- all the indices are positive: $j_{1}, \ldots, j_{k}, i_{1}, \ldots, i_{k} \geq 1$.

$-j$ and $i$ are ordered: $j_{1} \geq \ldots \geq j_{k}$ and $i_{1} \geq \ldots \geq i_{k}$.

We begin with two technical lemmas whose proofs are postponed at the end of this proof.

Lemma 2.19. - There is a constant $C>0$ such that for any $j \in \overline{\mathbb{Z}}^{k_{1}}$, $i \in \overline{\mathbb{Z}}^{k_{2}}$ and $l \in \overline{\mathbb{Z}}$ we have

$$
A(j, l)^{2} A(i, l)^{2} \leq C A(i, j)
$$


Lemma 2.20. - There is a constant $C>0$ such that for any $j \in \overline{\mathbb{Z}}^{k_{1}}$, $i \in \overline{\mathbb{Z}}^{k_{2}}$ and $l \in \overline{\mathbb{Z}}$ we have

$$
\max \left(\mu(j, l) A(i, l)^{2}, \mu(i, l) A(j, l)^{2}\right) \leq C \mu(i, j)^{2} .
$$

Using these lemmas, in order to prove (2.19), it suffices to prove

$$
\sum_{l \in \overline{\mathbb{Z}}} \frac{1}{C(j, l)^{\beta}(1+S(j, l))} \frac{A(i, l)^{2}}{C(i, l)^{\beta}} \leq C \frac{\mu(j, i)}{C(i, j)^{\beta}} .
$$

Noticing that $C(i, l) C(j, l) \geq C(i, j) l$, it suffices to verify that

$$
\sum_{l \in \overline{\mathbb{Z}}} \frac{A(i, l)^{2}}{(1+S(j, l)) l^{\beta}} \leq C \mu(j, i) .
$$

Decompose the sum in two parts, $I_{1}=\sum_{l>j_{2}}$ and $I_{2}=\sum_{l \leq j_{2}}$. For the first sum we have

$$
I_{1}=\sum_{l>j_{2}} \frac{A(i, l)^{2}}{(1+S(j, l)) l^{\beta}} \leq \sum_{l \in \overline{\mathbb{Z}}} \frac{1}{\left(1+\left|l-j_{1}\right|\right) l^{\beta}} \leq C,
$$

while for the second one

$$
I_{2}=\sum_{l \leq j_{2}} \frac{A(i, l)^{2}}{(1+S(j, l)) l^{\beta}} \leq \sum_{l \leq j_{2}} \frac{A(i, l)^{2}}{l^{\beta}} .
$$

In this last sum, if $j_{2}<\mu(i, j)$, then

$$
I_{2} \leq j_{2} \leq \mu(i, j) .
$$

On the other hand, if $\mu(i, j) \leq j_{2}$, then we decompose the $I_{2}$ sum in two parts, $I_{2,1}=\sum_{l<2 i_{1}}$ and $I_{2,2}=\sum_{l \geq 2 i_{1}}$. Since $i_{1} \leq \mu(i, j)=\max \left(i_{1}, j_{3}\right)$ we have

$$
I_{2,1}=\sum_{l \leq 2 i_{1}} \frac{A(i, l)^{2}}{l^{\beta}} \leq 2 i_{1} \leq 2 \mu(i, j) .
$$

Finally, when $l \geq 2 i_{1}$ we have $S(i, l) \geq l / 2$ and $B(i, l)^{2}=i_{1} i_{2} \leq i_{2} l / 2 \leq$ $\mu(i, j) l / 2$ and thus $A(i, l) \leq \sqrt{2 \mu(i, j)} l^{-1 / 2}$ which leads to

$$
I_{2,2}=\sum_{2 i_{1} \leq l \leq j_{2}} \frac{A(i, l)^{2}}{l^{\beta}} \leq C \mu(i, j) \sum_{l \in \overline{\mathbb{N}}} \frac{1}{l^{1+\beta}} \leq C \mu(i, j) .
$$

Proof of lemma 2.19 - The estimate (2.20), being symmetric with respect to $i$ and $j$, we can assume that $j_{1} \geq i_{1}$. We consider three cases, depending of the position of $l$ with respect to $i_{1}$ and $j_{1}$.

First case $l \geq j_{1}$ : 
We have $S(i, l)=\left|i_{1}-l\right| \geq\left|i_{1}-j_{1}\right| \geq S(i, j)$ and $B(i, l)=\left(i_{1} i_{2}\right)^{1 / 2} \leq B(i, j)$. Therefore

$$
A(i, l)=\frac{B(i, l)}{B(i, l)+S(i, l)} \leq \frac{B(i, l)}{B(i, l)+S(i, j)} \leq \frac{B(i, j)}{B(i, j)+S(i, j)}=A(i, j),
$$

and using $A(j, l) \leq 1,(2.20)$ is proved.

Second case $l \leq i_{1}$ :

Similarly as in the first case, we have $S(j, l) \geq S(i, j)$ and $B(j, l)=$ $\left(j_{2} \max \left(j_{3}, l\right)\right)^{1 / 2} \leq\left(j_{2} \max \left(j_{3}, i_{1}\right)\right)^{1 / 2} \leq B(i, j)$ and thus

$$
A(j, l) \leq A(i, j) .
$$

Third case $i_{1}<l<j_{1}$ :

That is the most complicated case and we have to distinguish whether $i_{1} \geq j_{2}$ or not.

Subcase 1. $i_{1} \geq j_{2}$ :

We have $B(i, l) \leq B(i, j)$ thus if $S(i, l)=\left|i_{1}-l\right| \geq \frac{1}{2}\left|i_{1}-j_{1}\right|=\frac{1}{2} S(i, j)$ we obtain $A(j, l) \leq 2 A(i, j)$ and (2.20) holds true. Now if $S(i, l)<\frac{1}{2} S(i, j)$ then $S(j, l) \geq \frac{1}{2} S(i, j)$ since $S(i, l)+S(j, l) \geq S(i, j)$. Furthermore, if $B(j, l) \leq$ $B(i, j)$ then

$$
A(j, l)=\frac{B(j, l)}{B(j, l)+S(j, l)} \leq 2 \frac{B(j, l)}{B(j, l)+S(i, j)} \leq 2 \frac{B(i, j)}{B(i, j)+S(i, j)}=2 A(i, j),
$$

and (2.20) holds. If $B(j, l)>B(i, j)$, then using

$B(j, l)^{2}=j_{2} l=j_{2} i_{1}+j_{2}\left(l-i_{1}\right) \leq B(i, j)^{2}+j_{2} S(i, l) \leq B(i, j)^{2}+\frac{1}{2} B(i, j) S(i, j)$,

we deduce

$A(j, l)^{2} \leq \frac{B(j, l)^{2}}{\left(B(i, j)+\frac{1}{2} S(i, j)\right)^{2}} \leq 2 \frac{B(i, j)^{2}+B(i, j) S(i, j)}{(B(i, j)+S(i, j))^{2}} \leq 2\left(A(i, j)^{2}+A(i, j)\right)$,

thus (2.20) is also satisfied in this case, since $A(i, j) \leq 1$.

Subcase 2. $i_{1} \leq j_{2}$ :

We still have $B(i, l) \leq B(i, j)$ thus if furthermore $S(i, j) \leq 2 S(i, l)$ then $A(i, l) \leq 2 A(i, j)$ and (2.20) is true. So we assume $2 S(i, l)<S(i, j)$ which implies $S(i, j) \leq 2 S(j, l)$ since $S(i, l)+S(j, l) \geq S(i, j)$. If furthermore $l \leq j_{3}$, $B(j, l)=B(j) \leq B(i, j)$ and thus $A(j, l) \leq 2 A(i, j)$ and (2.20) is again true. So we assume $j_{3} \leq l$ and we have

$$
B(j, l)^{2}=l j_{2}=i_{1} j_{2}+j_{2}\left(l-i_{1}\right) \leq B(i, j)^{2}+j_{2} S(i, l) .
$$


If $S(i, l) \leq l / 2$ then we deduce $B(j, l)^{2} \leq 2 B(j, l)^{2}$ and (2.20) is satisfied. It remains to consider the case $S(i, l)>l / 2$ which implies $i_{1}<l / 2$ and thus

$$
A(i, l) \leq \frac{i_{1}}{i_{1}+l / 2} \leq 2 \frac{i_{1}}{l} .
$$

Let $n \geq 1$ such that $\frac{l}{2^{n+1}} \leq i_{1} \leq \frac{l}{2^{n}}$ we get from (2.22)

$$
A(i, l) \leq \frac{1}{2^{n-1}} .
$$

On the other hand

$$
A(j, l) \leq 2 \frac{\left(l j_{2}\right)^{1 / 2}}{\left(l j_{2}\right)^{1 / 2}+S(i, j)} \leq 2 \frac{\left(l j_{2}\right)^{1 / 2}}{\left(i_{1} j_{2}\right)^{1 / 2}+S(i, j)}
$$

and

$$
A(i, j) \geq \frac{\left(i_{1} j_{2}\right)^{1 / 2}}{\left(i_{1} j_{2}\right)^{1 / 2}+S(i, j)} \geq \frac{1}{2^{n+1}} \frac{\left(l j_{2}\right)^{1 / 2}}{\left(i_{1} j_{2}\right)^{1 / 2}+S(i, j)} .
$$

Combining (2.23), (2.24) and (2.25) we conclude

$$
A(i, l) A(j, l) \leq 8 A(i, j) .
$$

Proof of lemma 2.20 - The estimate (2.21) being symmetric with respect to $i$ and $j$, we can assume $j_{1} \geq i_{1}$. If furthermore $i_{1} \geq j_{2}$ then one easily verifies that

$$
\mu(i, l) \leq \mu(i, j) \text { and } \mu(j, l) \leq \mu(i, j)
$$

and estimates (2.21) is satisfied.

In the case $j_{1} \geq j_{2} \geq i_{1}$ we still have $\mu(i, l) \leq \mu(i, j)$ but $\mu(j, l)$ could be larger than $\mu(i, j)$. Actually if $\mu(j, l) \leq 2 \mu(i, j)$, estimates (2.21) is still trivially satisfied. Therefore it remains to consider the case where $\mu(j, l)>2 \mu(i, j)$. Remark that in this case $i_{1} \leq \mu(i, j) \leq \frac{\mu(j, l)}{2} \leq l / 2$ and thus $S(i, l)=\left|i_{1}-l\right| \geq$ $l / 2$ which leads to

$$
A(i, l) \leq \frac{\left(i_{1} i_{2}\right)^{1 / 2}}{S(i, l)} \leq \frac{\left(2 i_{2}\right)^{1 / 2}}{l^{1 / 2}} \leq \frac{(2 \mu(i, j))^{1 / 2}}{l^{1 / 2}} .
$$

Using this last estimates one gets

$$
\mu(j, l) A(i, l)^{2} \leq l A(i, l)^{2} \leq 2 \mu(i, j)^{2} .
$$

We end this section with a proposition concerning Lie transforms of homogeneous polynomials $\chi \in \mathcal{T}_{l}^{\delta, \beta,+}$, i.e. time 1 flow of the Hamiltonian vector field $X_{\chi}$. 
Proposition 2.21. - Let $\chi$ be a real homogeneous polynomial in $\mathcal{T}_{l}^{\delta, \beta,+}$ with $\delta \geq 0, \beta>0, l \geq 3$ take $s>s_{1}:=\delta+3 / 2$ and denote by $\phi$ the Lie transform associated with $\chi$. We have

(i) $\phi$ is an analytic canonical transformation from an open ball $B_{\epsilon}$ of center 0 and radius $\epsilon$ in $\mathcal{P}_{s}$ into the open ball $B_{2 \epsilon}$ in $\mathcal{P}_{s}$ satisfying

$$
\|\phi(z)-z\|_{s} \leq C_{s}\|z\|_{s}^{2} \text { for any } z \in B_{\varepsilon} .
$$

In particular if $F \in \mathcal{H}^{s}$ with $s>s_{1}$ then $F \circ \phi \in \mathcal{H}^{s}$. Furthermore, if $F$ is real then $F \circ \phi$ is real too.

(ii) Let $P \in \mathcal{T}_{n}^{\nu, \beta} \cap \mathcal{H}^{s}, \nu \geq 0, n \geq 3$ and fix $r \geq n$ an integer. Then

$$
P \circ \phi=Q_{r}+R_{r}
$$

where:

- $Q_{r}$ is a polynomial of degree at most $r$, belonging to $\mathcal{T}^{\nu^{\prime}, \beta} \cap \mathcal{H}^{s}$ with $\nu^{\prime}=2^{r-n} \nu+\left(2^{r-n}-1\right)(2 \beta+1)$,

- $R_{r}$ is a Hamiltonian in $\mathcal{T}^{\nu^{\prime \prime}, \beta} \cap \mathcal{H}^{s}$ with $\nu^{\prime \prime}=2^{r-n+1} \nu+\left(2^{r-n+1}-\right.$ $1)(2 \beta+1)$, having a zero of order $r+1$ at the origin.

Proof. - (i) Since $\chi \in \mathcal{T}_{l}^{\delta, \beta,+}$, by Proposition 2.13(iii), $X_{\chi} \in C^{\infty}\left(\mathcal{P}_{s}, \mathcal{P}_{s}\right)$ for $s>s_{1}=\delta+3 / 2$. In particular, for $s>s_{1}$, the flow $\Phi^{t}$ generated by the vector field $X_{\chi}$ transports an open neighborhood of the origin in $\mathcal{P}_{s}$ into an open neighborhood of the origin in $\mathcal{P}_{s}$. Notice that since $\chi$ is real, $\Phi^{t}$ transports the "real part" of $\mathcal{P}_{s},\left\{(\xi, \bar{\xi}) \in \mathcal{P}_{s}\right\}$, into itself. Furthermore one has for $z \in \mathcal{P}_{s}$ small enough

$$
\Phi^{t}(z)-z=\int_{0}^{t} X_{\chi}\left(\Phi^{t^{\prime}}(z)\right) d t^{\prime}
$$

and since $\chi$ has a zero of order 3 at least, one gets by Proposition 2.13(iii),

$$
\left\|\Phi^{t}(z)-z\right\|_{s} \leq C_{s} \int_{0}^{t}\left\|\Phi_{\chi}^{t^{\prime}}(z)\right\|_{s}^{2} d t^{\prime}
$$

Then, by a classical continuity argument, there exists $\epsilon>0$ such that the flow $B_{\varepsilon} \ni z \mapsto \Phi_{\chi}^{t}(z) \in B_{2 \varepsilon}$ is well defined and smooth for $0 \leq t \leq 1$. Furthermore, the Lie transform $\phi=\Phi^{1}$ satisfies (2.26).

On the other hand, by simple composition we get that if $F \in \mathcal{H}^{s}$ with $s>s_{1}$, then $F \circ \phi \in C^{\infty}\left(B_{\epsilon}, \mathbb{C}\right)$. In view of the formula

$$
X_{F \circ \phi}(z)=(D \phi(z))^{-1} X_{F}(\phi(z)),
$$

we deduce that $X_{F \circ \phi} \in C^{\infty}\left(B_{\epsilon}, \mathcal{P}_{s}\right)$. We now have to check the properties concerning the Taylor polynomials of $F \circ \phi$. Denoting by $F_{k}\left(\right.$ resp. $\left.(F \circ \phi)_{k}\right)$ 
the homogeneous polynomial of degree $k$ appearing in the Taylor expansion of $F(\operatorname{resp} F \circ \phi)$, and putting $F_{k}^{[0]}=F_{k}, F_{k}^{[j+1]}=\left\{F_{k}, \chi\right\}$, we have

$$
(F \circ \phi)_{k}(z)=\sum_{j \geq 0, k^{\prime} \geq 0, k^{\prime}+j(l-2)=k} F_{k^{\prime}}^{[j]}(z),
$$

since $\chi$ is itself a homogeneous polynomial of degree $l$. It is then sufficient to prove that the Poisson bracket of a homogeneous polynomial $F_{k}$ in $\mathcal{H}^{s}$ with $\chi$ stays in $\mathcal{H}^{s}$.

Using the (constant) symplectic form $\omega$ on $\mathcal{P}_{s}$, we get $\left\{F_{k}, \chi\right\}(z)=$ $\omega\left(X_{F_{k}}, X_{\chi}\right)$, and so $\left\{F_{k}, \chi\right\} \in C^{\infty}\left(B_{\epsilon}, \mathbb{C}\right)$. Moreover

$$
X_{\left\{F_{k}, \chi\right\}}(z)=\left[X_{F_{k}}, X_{\chi}\right]=\lim _{t \rightarrow 0} \frac{1}{t}\left(X_{F_{k}}-\Phi_{*}^{t}\left(X_{F_{k}}\right)\right)(z) .
$$

Since $\Phi^{t}$ is the flow of the regular Hamiltonian $\chi \in C^{\infty}\left(B_{\epsilon}, \mathbb{R}\right)$, the Cauchy Lipschitz theorem implies that the mapping $(t, z) \mapsto \Phi_{*}^{t}\left(X_{F_{k}}\right)(z)$ is in $C^{\infty}\left([-1,1] \times B_{\epsilon}, \mathcal{P}_{s}\right)$. Now, $X_{\left\{F_{k}, \chi\right\}}$ is nothing else but the time derivative of this mapping at time 0 , hence $X_{\left\{F_{k}, \chi\right\}} \in C^{\infty}\left(B_{\epsilon}, \mathcal{P}_{s}\right)$ and the claim is proved.

(ii) By a direct calculus one has

$$
\left.\frac{d^{k}}{d t^{k}} P \circ \Phi^{t}(z)\right|_{t=0}=P^{[k]}(z)
$$

with the same notation $P^{[k+1]}=\left\{P^{[k]}, \chi\right\}$ and $P^{[0]}=P$. Therefore applying the Taylor's formula to $P \circ \Phi^{t}(z)$ between $t=0$ and $t=1$ we deduce

$$
P \circ \phi(z)=\sum_{k=0}^{r-n} \frac{1}{n !} P^{[k]}(z)+\frac{1}{(r-n) !} \int_{0}^{1}(1-t)^{r} P^{[r-n+1]}\left(\Phi^{t}(z)\right) d t .
$$

Notice that $P^{[k]}(z)$ is a homogeneous polynomial of degree $n+k(l-2)$ and, by Proposition [2.18, $P^{[k]}(z) \in \mathcal{T}^{2^{k} \nu+\left(2^{k}-1\right)(2 \delta+1), \beta}$. Moreover $P^{[k]}(z)$ is a homogeneous polynomial in the Taylor expansion of $P \circ \phi \in \mathcal{H}^{s}$, hence it is in $\mathcal{H}^{s}$. Therefore (2.27) decomposes in the sum of a polynomial of degree $r$ in $\mathcal{T}_{r}^{\nu^{\prime}, \beta}$, and a function in $\mathcal{H}^{s}$ having a zero of degree $r+1$ at the origin.

2.3. The Birkhoff normal form theorem. - We start with the resolution of the homological equation and then state the normal form theorem.

Lemma 2.22. - Let $\nu \in[0,+\infty)$ and assume that the frequency vector of $H_{0}$ is strongly non resonant (see Definition 2.5). Let $Q$ be a homogeneous real polynomial of degree $k$ in $\mathcal{T}_{k}^{\nu, \beta}$, there exist $\nu^{\prime}>\nu$, and $Z$ and $\chi$ two 
homogeneous real polynomials of degree $k$, respectively in $\mathcal{T}_{k}^{\nu^{\prime}, \beta}$ and $\mathcal{T}_{k}^{\nu^{\prime}, \beta,+}$, which satisfy

$$
\left\{H_{0}, \chi\right\}+Q=Z
$$

and

$$
\left\{Z, I_{j}\right\}=0 \quad \forall j \geq 1
$$

and thus $Z$ is in normal form. Furthermore, $Z$ and $\chi$ both belong to $\mathcal{H}^{s}$ for $s>\nu^{\prime}+1$.

Proof. - For $j \in \overline{\mathbb{N}}^{k_{1}}$ and $l \in \overline{\mathbb{N}}^{k_{2}}$ with $k_{1}+k_{2}=k$ we denote

$$
\xi^{(j)} \eta^{(l)}=\xi_{j_{1}} \ldots \xi_{j_{k_{1}}} \eta_{l_{1}} \ldots \eta_{l_{k_{2}}} \text {. }
$$

One has

$$
\left\{H_{0}, \xi^{(j)} \eta^{(l)}\right\}=-i \Omega(j, l) \xi^{(j)} \eta^{(l)}
$$

with

$$
\Omega(j, l):=\omega_{j_{1}}+\ldots+\omega_{j_{k_{1}}}-\omega_{l_{1}}-\ldots-\omega_{l_{k_{2}}} .
$$

Let $Q \in \mathcal{T}_{k}^{\nu, \beta}$

$$
Q=\sum_{(j, l) \in \overline{\mathbb{N}}^{k}} a_{j l} \xi^{(j)} \eta^{(l)}
$$

where $(j, l) \in \overline{\mathbb{N}}^{k}$ means that $j \in \overline{\mathbb{N}}^{k_{1}}$ and $l \in \overline{\mathbb{N}}^{k_{2}}$ with $k_{1}+k_{2}=k$. Let us define

$$
b_{j l}=i \Omega(j, l)^{-1} a_{j l}, \quad c_{j l}=0 \quad \text { when }\left\{j_{1}, \ldots, j_{k_{1}}\right\} \neq\left\{l_{1}, \ldots, l_{k_{2}}\right\}
$$

and

$$
c_{j l}=a_{j l}, \quad b_{j l}=0 \quad \text { when }\left\{j_{1}, \ldots, j_{k_{1}}\right\}=\left\{l_{1}, \ldots, l_{k_{2}}\right\} .
$$

As $\omega$ is strongly non resonant, there exist $\gamma$ and $\alpha$ such that

$$
|\Omega(j, l)| \geq \gamma \frac{1+S(j, l)}{\mu(j, l)^{\alpha}}
$$

for all $(j, l) \in \overline{\mathbb{N}}^{k}$ with $\left\{j_{1}, \ldots, j_{k_{1}}\right\} \neq\left\{l_{1}, \ldots, l_{k_{2}}\right\}$. Thus, in view of Definitions 2.6 and 2.7, the polynomial

$$
\chi=\sum_{(j, l) \in \overline{\mathbb{N}} k} b_{j, l} \xi^{(j)} \eta^{(l)},
$$

belongs to $\mathcal{T}_{k}^{\nu^{\prime}, \beta,+}$ while the polynomial

$$
Z=\sum_{(j, l) \in \overline{\mathbb{N}}^{k}} c_{j, l} \xi^{(j)} \eta^{(l)}
$$


belongs to $\mathcal{T}_{k}^{\nu^{\prime}, \beta}$ with $\nu^{\prime}=\nu+\alpha$. Notice that in this non resonant case, (2.29) implies that $Z$ depends only on the actions and thus is in normal form. Furthermore by construction they satisfy (2.28) and (2.29). Note that the reality of $Q$ is equivalent to the symmetry relation: $\bar{a}_{j l}=a_{l j}$. Taking into account that $\Omega_{l j}=-\Omega_{j l}$, this symmetry remains satisfied for the polynomials $\chi$ and $Z$. Finally, $\chi$ and $Z$ belong to $\mathcal{H}^{s}$, since they are homogeneous polynomials (they are their own Taylor expansions) and as a consequence of Proposition 2.13 (iii) and (iv) respectively.

We can now state the main result of this section:

Theorem 2.23. - Assume that $P$ is a real Hamiltonian belonging to $\mathcal{H}^{s}$ for all $s$ large enough and to the class $\mathcal{T}^{\nu, \beta}$ for some $\nu \geq 0$ and $\beta>0$. Assume that $\omega$ is strongly non resonant (cf. Definition [2.5) and satisfies (2.1) for some $\bar{d} \geq 0$. Then for any $r \geq 3$ there exists $s_{0}$ and for any $s \geq s_{0}$ there exists $\mathcal{U}_{s}, \mathcal{V}_{s}$ neighborhoods of the origin in $\mathcal{P}_{s}$ and $\tau_{s}: \mathcal{V}_{s} \rightarrow \mathcal{U}_{s}$ a real analytic canonical transformation which is the restriction to $\mathcal{V}_{s}$ of $\tau:=\tau_{s_{0}}$ and which puts $H=H_{0}+P$ in normal form up to order $r$ i.e.

$$
H \circ \tau=H_{0}+Z+R
$$

with

(a) $Z$ is a real continuous polynomial of degree $r$ with a regular vector field (i.e. $Z \in \mathcal{H}^{s}$ ) which only depends on the actions: $Z=Z(I)$.

(b) $R \in \mathcal{H}^{s}$ is real and $\left\|X_{R}(z)\right\|_{s} \leq C_{s}\|z\|_{s}^{r}$ for all $z \in \mathcal{V}_{s}$.

(c) $\tau$ is close to the identity: $\|\tau(z)-z\|_{s} \leq C_{s}\|z\|_{s}^{2}$ for all $z \in \mathcal{V}_{s}$.

Proof. - The proof is close to the proof of Birkhoff normal form theorem stated in Gré07] or [Bam07. The main difference has been already pointed out : we have here to check the $\mathcal{H}^{s}$ regularity of the Hamiltonian functions at each step, independently of the fact that they belong to $\mathcal{T}^{\nu, \beta}$ (here $P \in \mathcal{T}^{\nu, \beta}$ does not imply $P \in \mathcal{H}^{s}$ ).

Having fixed some $r \geq 3$, the idea is to construct iteratively for $k=3, \ldots, r$, a neighborhood $\mathcal{V}_{k}$ of 0 in $\mathcal{P}_{s}$ ( $s$ large enough depending on $r$ ), a canonical transformation $\tau_{k}$, defined on $\mathcal{V}_{k}$, an increasing sequence $\left(\nu_{k}\right)_{k=3, \ldots, r}$ of positive numbers and real Hamiltonians $Z_{k}, P_{k+1}, Q_{k+2}, R_{k}$ such that

$$
H_{k}:=H \circ \tau_{k}=H_{0}+Z_{k}+P_{k+1}+Q_{k+2}+R_{k},
$$

satisfying the following properties 
(i) $Z_{k}$ is a polynomial of degree $k$ in $\mathcal{T}^{\nu_{k}, \beta} \cap \mathcal{H}^{s}$ having a zero of order 3 (at least) at the origin and $Z_{k}$ depends only on the (new) actions: $\left\{Z_{k}, I_{j}\right\}=0$ for all $j \geq 1$.

(ii) $P_{k+1}$ is a homogeneous polynomial of degree $k+1$ in $\mathcal{T}_{k+1}^{\nu_{k}, \beta} \cap \mathcal{H}^{s}$.

(iii) $Q_{k+2}$ is a polynomial of degree $r+1$ in $\mathcal{T}^{\nu_{k}, \beta} \cap \mathcal{H}^{s}$ having a zero of order $k+2$ at the origin.

(iv) $R_{k}$ is a regular Hamiltonian belonging to $\mathcal{H}^{s}$ and having a zero of order $r+2$ at the origin.

First we fix $s>\nu_{r}+3 / 2$ to be sure to be able to apply Proposition 2.13 at each step $\left(\nu_{r}\right.$ will be defined later on independently of $\left.s\right)$. Then we notice that (2.32) at order $r$ proves Theorem 2.23 with $Z=Z_{r}$ and $R=P_{r+1}+R_{r}$ (since $\left.Q_{r+2}=0\right)$. Actually, since $R=P_{r+1}+R_{r}$ belongs to $\mathcal{H}^{s}$ and has a zero of order $r+1$ at the origin, we can apply Lemma 2.3 to obtain

$$
\left\|X_{R}(z)\right\|_{s} \leq C_{s}\|z\|_{s}^{r} .
$$

on $\mathcal{V} \subset \mathcal{V}_{r}$ a neighborhood of 0 in $\mathcal{P}_{s}$.

At the initial step (which for convenience we will denote the $k=2$ step), the Hamiltonian $H=H_{0}+P$ has the desired form (2.32) with $\tau_{2}=I, \nu_{2}=\nu$, $Z_{2}=0, P_{3}$ being the Taylor polynomial of $P$ of degree $3, Q_{4}$ being the Taylor polynomial of $P$ of degree $r+1$ minus $P_{3}$ and $R_{2}=P-P_{3}-Q_{4}$. We show now how to go from step $k$ to step $k+1$.

We look for $\tau_{k+1}$ of the form $\tau_{k} \circ \phi_{k+1}, \phi_{k+1}$ being the Lie transform associated to a homogeneous polynomial $\chi_{k+1}$ of degree $k+1$.

We decompose $H_{k} \circ \phi_{k+1}$ as follows

$$
\begin{aligned}
H_{k} \circ \phi_{k+1} & =H_{0}+Z_{k}+\left\{H_{0}, \chi_{k+1}\right\}+P_{k+1} \\
& +H_{0} \circ \phi_{k+1}-H_{0}-\left\{H_{0}, \chi_{k+1}\right\} \\
& +Z_{k} \circ \phi_{k+1}-Z_{k} \\
& +P_{k+1} \circ \phi_{k+1}-P_{k+1} \\
& +Q_{k+2} \circ \phi_{k+1} \\
& +R_{k} \circ \phi_{k+1} .
\end{aligned}
$$

Using Lemma 2.22 above, we choose $\chi_{k+1}$ in $\mathcal{T}_{k+1}^{\nu_{k}^{\prime}, \beta,+}$ in such a way that

$$
\hat{Z}_{k+1}:=\left\{H_{0}, \chi_{k+1}\right\}+P_{k+1}
$$

is a homogeneous real polynomial of degree $k+1$ in $\mathcal{T}_{k+1}^{\nu_{k}^{\prime}, \beta}$. We put then $Z_{k+1}=Z_{k}+\hat{Z}_{k+1}$, which obviously has degree $k+1$ and a zero of order 3 (at least) at the origin, and the right hand side of line (2.34) becomes $H_{0}+Z_{k+1}$. 
We just recall that $\nu_{k}^{\prime}=\nu_{k}+\alpha$, where $\alpha$ is determined by $\omega$, independently of $r$ and $s$. By Proposition 2.21, the Lie transform associated to $\chi_{k+1}$ is well defined and smooth on a neighborhood $\mathcal{V}_{k+1} \subset \mathcal{V}_{k}$ and, for $z \in \mathcal{V}_{k+1}$ satisfies

$$
\left\|\phi_{k+1}(z)-z\right\|_{s} \leq C\|z\|_{s}^{2} .
$$

Then from Proposition 2.18, Proposition 2.21 and formula (2.27), we find that (2.36), (2.37), (2.38) and (2.39) are regular Hamiltonians having zeros of order $k+2$ at the origin. For instance concerning (2.36), one has by Taylor formula for any $z \in \mathcal{V}_{k+1}$

$Z_{k} \circ \phi_{k+1}(z)-Z_{k}(z)=\left\{Z_{k}, \chi_{k+1}\right\}(z)+\int_{0}^{1}(1-t)\left\{\left\{Z_{k}, \chi_{k+1}\right\}, \chi_{k+1}\right\}\left(\Phi_{\chi_{k+1}}^{t}(z)\right) d t$

and $\left\{Z_{k}, \chi_{k+1}\right\}$ is a polynomial having a zero of order $3+\operatorname{degree}\left(\chi_{k+1}\right)-2=$ $k+2$ while the integral term is a regular Hamiltonian having a zero of order $2 k+1$. Thus if $2 k+1 \geq r+2$ this last term contributes to $R_{k+1}$ and if not, we have to use a Taylor formula at a higher order.

Therefore the sum of (2.36), (2.37), (2.38) and (2.39) decomposes in $\tilde{P}_{k+2}+$ $\tilde{Q}_{k+3}+\tilde{R}_{k+1}$ with $\tilde{P}_{k+2}, \tilde{Q}_{k+3}$ and $\tilde{R}_{k+1}$ satisfying respectively the properties (ii), (iii) and (iv) at rank $k+1$ (with $\nu_{k+1}=k \nu_{k}^{\prime}+\nu_{k}+k+2$ ).

Concerning the term (2.35), one has to proceed differently since $H_{0}$ does not belong to the $\mathcal{H}^{s}$.

First notice that by the homological equation (2.40) one has $\left\{H_{0}, \chi_{k+1}\right\}=$ $Z_{k+1}-Z_{k}-P_{k+1}$. By construction $Z_{k}$ and $P_{k+1}$ belong to $\mathcal{H}^{s}$. On the other hand, by Lemma 2.22, $Z_{k+1} \in \mathcal{T}_{k+1}^{\nu_{k}^{\prime}, \beta}$ and is in normal form (i.e. it depends only on the action variables). Thus by Proposition 2.13 assertion (iv), one concludes that $Z_{k+1} \in \mathcal{H}^{s}$. Therefore we have proved that $\left\{H_{0}, \chi_{k+1}\right\} \in \mathcal{H}^{s}$. Now we use the Taylor formula at order one to get

$$
H_{0} \circ \phi_{k+1}(z)-H_{0}(z)=\int_{0}^{1}\left\{H_{0}, \chi_{k+1}\right\}\left(\Phi_{\chi_{k+1}}^{t}(z)\right) d t .
$$

But we know from the proof of Proposition 2.21 that $\Phi_{\chi_{k+1}}^{t}: \mathcal{V}_{k+1} \rightarrow \mathcal{P}_{s}$ for all $t \in[0,1]$. Therefore $H_{0} \circ \phi_{k+1}-H_{0} \in \mathcal{H}^{s}$ and thus (2.35) defines a regular Hamiltonian.

Finally we use again the Taylor formula and the homological equation to write

$$
\begin{aligned}
H_{0} \circ \phi_{k+1}(z)-H_{0}(z)-\{ & \left.H_{0}, \chi_{k+1}\right\}(z)= \\
& \int_{0}^{1}(1-t)\left\{Z_{k+1}-Z_{k}-P_{k+1}, \chi_{k+1}\right\}\left(\Phi_{\chi_{k+1}}^{t}(z)\right) d t
\end{aligned}
$$


and , since $Z_{k+1}-Z_{k}-P_{k+1}$ belongs to $\mathcal{T}_{k+1}^{\nu_{k}^{\prime}, \beta}$ and $\chi_{k+1} \in \mathcal{T}_{k+1}^{\nu_{k}^{\prime}, \beta,+}$ we conclude by Proposition 2.18 that $H_{0} \circ \phi_{k+1}-H_{0}-\left\{H_{0}, \chi_{k+1}\right\} \in \mathcal{T}^{\nu_{k+1}, \beta}$. Finally we use Proposition 2.21 to decompose it in $\hat{P}_{k+2}+\hat{Q}_{k+3}+\hat{R}_{k+1}$ with $\hat{P}_{k+2}, \hat{Q}_{k+3}$ and $\hat{R}_{k+1}$ satisfying respectively the properties (ii), (iii) and (iv) at rank $k+1$. The proof is achieved defining $P_{k+2}=\hat{P}_{k+2}+\tilde{P}_{k+2}, Q_{k+3}=\hat{Q}_{k+3}+\tilde{Q}_{k+3}$ and $R_{k+1}=\hat{R}_{k+1}+\tilde{R}_{k+1}$.

\section{Dynamical consequences}

3.1. Nonlinear harmonic oscillator in one dimension. - We recall the notations of the introduction. The quantum harmonic oscillator $T=-\frac{d^{2}}{d x^{2}}+x^{2}$ is diagonalized in the Hermite basis $\left(\phi_{j}\right)_{j \in \overline{\mathbb{N}}}$ :

$$
\begin{aligned}
T \phi_{j} & =(2 j-1) \phi_{j}, \quad j \in \overline{\mathbb{N}} \\
\phi_{n+1} & =\frac{H_{n}(x)}{\sqrt{2^{n} n !}} e^{-x^{2} / 2}, \quad n \in \mathbb{N}
\end{aligned}
$$

where $H_{n}(x)$ is the $n^{\text {th }}$ Hermite polynomial relative to the weight $e^{-x^{2}}$ :

$$
\int_{\mathbb{R}} e^{-x^{2}} H_{m}(x) H_{n}(x) d x=2^{n} n ! \sqrt{\pi} \delta_{n m} .
$$

In this basis, the Hermite multiplier is given by

$$
M \phi_{j}=m_{j} \phi_{j}
$$

where $\left(m_{j}\right)_{j \in \overline{\mathbb{N}}}$ is a bounded sequence of real number. For any $k \geq 1$, we define the class

$$
\mathcal{W}_{k}=\left\{\left(m_{j}\right)_{j \in \overline{\mathbb{N}}} \mid \text { for each } j, m_{j}=\frac{\tilde{m}_{j}}{j^{k}} \text { with } \tilde{m}_{j} \in[-1 / 2,1 / 2]\right\}
$$

that we endow with the product probability measure. In this context the frequencies, i.e. the eigenvalues of $T+M=-d^{2} / d x^{2}+x^{2}+M$ are given by

$$
\omega_{j}=2 j-1+m_{j}=2 j-1+\frac{\tilde{m}_{j}}{j^{k}}, \quad j \in \overline{\mathbb{N}} .
$$

Proposition 3.1. - There exists a set $F_{k} \subset \mathcal{W}_{k}$ whose measure equals 1 such that if $m=\left(m_{j}\right)_{j \in \overline{\mathbb{N}}} \in F_{k}$ then the frequency vector $\left(\omega_{j}\right)_{j \geq 1}$ is strongly non-resonant (cf. Definition 2.5).

Proof. - First remark that it suffices to prove that the frequency vector $\left(\omega_{j}\right)_{j \geq 1}$ is non resonant in the sense of Definition 2.4. Actually, if we prove 
that (2.3) is satisfied for given constants $\delta^{\prime}$ and $\gamma^{\prime}$ then if $S(j)<r \mu(j)$

$$
\left|\omega_{j_{1}}+\cdots+\omega_{j_{i}}-\omega_{j_{i+1}}-\cdots-\omega_{j_{r}}\right| \geq \frac{\gamma^{\prime}}{\mu(j)^{\delta^{\prime}}} \geq \frac{\gamma^{\prime}}{r+1} \frac{1+S(j)}{\mu(j)^{\delta^{\prime}+1}}
$$

and thus (2.4) is satisfied with $\delta=\delta^{\prime}+1$ and $\gamma=\frac{\gamma^{\prime}}{r+1}$. Now if $S(j) \geq r \mu(j)$ then use

$$
\left|\omega_{j_{1}}+\cdots+\omega_{j_{i}}-\omega_{j_{i+1}}-\cdots-\omega_{j_{r}}\right| \geq S(j)-(r-2) \mu(j),
$$

to conclude that

$$
\left|\omega_{j_{1}}+\cdots+\omega_{j_{i}}-\omega_{j_{i+1}}-\cdots-\omega_{j_{r}}\right| \geq \frac{2}{r} S(j) \geq \frac{\gamma^{\prime}}{r+1} \quad \frac{1+S(j)}{\mu(j)^{\delta^{\prime}+1}}
$$

provided $\gamma^{\prime}$ is small enough.

The proof that there exists a set $F_{k} \subset \mathcal{W}_{k}$ whose measure equals 1 such that if $m=\left(m_{j}\right)_{j \in \overline{\mathbb{N}}} \in F_{k}$ then the frequency vector $\left(\omega_{j}\right)_{j \geq 1}$ is non resonant is exactly the same as the proof of Theorem 5.7 in [Gré07]. So we do not repeat it here (see also [BG06]).

In equation (1.1) with $d=1$, the Hamiltonian perturbation reads

$$
P(\xi, \eta)=\int_{\mathbb{R}} g(\xi(x), \eta(x)) d x
$$

where $g \in C^{\infty}\left(\mathbb{C}^{2}, \mathbb{C}\right), \xi(x)=\sum_{j \geq 1} \xi_{j} \phi_{j}(x), \eta(x)=\sum_{j \geq 1} \eta_{j} \phi_{j}(x)$ and $\left(\left(\xi_{j}\right)_{j \geq 1},\left(\eta_{j}\right)_{j \geq 1}\right) \in \mathcal{P}_{s}$. We first check that $P$ belongs to $\mathcal{H}^{s}$ for $s$ large enough.

Lemma 3.2. - Let $P$ given by (3.4) with $g \in C^{\infty}(\mathcal{U}, \mathbb{C}), \mathcal{U}$ being a neighborhood of 0 in $\mathbb{C}^{2}, g$ real i.e. $g(z, \bar{z}) \in \mathbb{R}$ and $g$ having a zero of order at least 3 at the origin. Then $P \in \mathcal{H}^{s}$ for all $s>1 / 2$.

Proof. — One computes

$$
\frac{\partial P}{\partial \xi_{j}}(\xi, \eta)=\int_{\mathbb{R}} \partial_{1} g(\xi(x), \eta(x)) \phi_{j}(x) d x
$$

and

$$
\frac{\partial P}{\partial \eta_{j}}(\xi, \eta)=\int_{\mathbb{R}} \partial_{2} g(\xi(x), \eta(x)) \phi_{j}(x) d x .
$$

In the same way, we have

$$
\begin{aligned}
& \frac{\partial^{l+r} P}{\partial \xi_{j_{1}} \ldots \partial \xi_{j_{l}} \partial \eta_{k_{1}} \ldots \partial \eta_{k_{r}}}(\xi, \eta) \\
& =\int_{\mathbb{R}} \partial_{1}^{l} \partial_{2}^{r} g(\xi(x), \eta(x)) \phi_{j_{1}}(x) \ldots \phi_{j_{l}}(x) \phi_{k_{1}}(x) \ldots \phi_{k_{r}}(x) d x .
\end{aligned}
$$


Since $g$ is a $C^{\infty}$ function, all these partial derivatives are continuous from $\mathcal{P}_{s}$ to $\mathbb{C}$, and the corresponding differentials $(\xi, \eta) \rightarrow D^{l+r} P(\xi, \eta)$ are continuous from $\mathcal{P}_{s}$ to the space of $l+r$-linear forms on $\mathcal{P}_{s}$. We get moreover

$$
\begin{aligned}
\left\|X_{P}(\xi, \eta)\right\|_{s}^{2} & =\sum_{j \geq 1}|j|^{2 s}\left|\int_{\mathbb{R}} \partial_{1} g(\xi(x), \eta(x)) \phi_{j}(x) d x\right|^{2} \\
& +\sum_{j \geq 1}|j|^{2 s}\left|\int_{\mathbb{R}} \partial_{2} g(\xi(x), \eta(x)) \phi_{j}(x) d x\right|^{2} .
\end{aligned}
$$

Therefore, to check that $z \mapsto X_{P}(z)$ is a regular function from a neighborhood of the origin in $\mathcal{P}_{s}$ into $\mathcal{P}_{s}$, it suffices to check that the functions $x \mapsto \partial_{1} g(\xi(x), \eta(x))$ and $x \mapsto \partial_{2} g(\xi(x), \eta(x))$ are in $\tilde{H}^{s}$ provided $\xi(x)$ and $\eta(x)$ are in $\tilde{H}^{s}$. So it remains to prove that functions of the type $x \mapsto|x|^{i} \partial_{1}^{l+1} \partial_{2}^{m} g(\xi(x), \eta(x))\left(\xi^{\left(l_{1}\right)}(x)\right)^{\alpha_{1}} \cdot\left(\xi^{\left(l_{k_{1}}\right)}(x)\right)^{\alpha_{k_{1}}}\left(\eta^{\left(m_{1}\right)}(x)\right)^{\beta_{1}} \cdots\left(\eta^{\left(m_{k_{2}}\right)}(x)\right)^{\beta_{k_{2}}}$ are in $L^{2}(\mathbb{R})$ for all $0 \leq i+l+m \leq s, 0 \leq i+l_{j} \leq s, 0 \leq i+m_{j} \leq s$. But this is true because

$-g$ is a $C^{\infty}$ function, $\xi$ and $\eta$ are bounded functions and thus $x \mapsto$ $\partial_{1}^{l+1} \partial_{2}^{m} g(\xi(x), \eta(x))$ is bounded

- $\tilde{H}^{s}$ is an algebra for $s>1 / 2$ and thus $x \mapsto|x|^{k} \xi^{(l)}(x) \eta^{(m)}(x) \in L^{2}(\mathbb{R})$ for all $0 \leq k+l+m \leq s$.

- $\left|\partial_{1} g(\xi(x), \eta(x))\right|,\left|\partial_{2} g(\xi(x), \eta(x))\right| \leq C(|\xi(x)|+|\eta(x)|)^{2}$ for some uniform constant $C>0$ and thus $x \mapsto|x|^{k} \partial_{1} g(\xi(x), \eta(x)) \in L^{2}(\mathbb{R})$ for all $0 \leq k \leq s$.

There remains to prove the same properties concerning the Taylor homogeneous polynomial $P_{m}$ of $P$ at any order $m$, computed at $(0,0)$. From (3.5), we get

$$
P_{m}=\frac{1}{m !} \int_{\mathbb{R}} \sum_{l+r=m} \partial_{1}^{l} \partial_{2}^{r} g(0,0) \sum_{j, k} \xi_{j_{1}} \phi_{j_{1}}(x) \ldots \xi_{j_{l}} \phi_{j_{l}}(x) \eta_{k_{1}} \phi_{k_{1}}(x) \ldots \eta_{k_{r}} \phi_{k_{r}}(x) d x,
$$

hence $P_{m}$ can be computed directly from formula (3.4), replacing $g$ by its Taylor homogeneous polynomial $g_{m}$ of order $m$ :

$$
g_{m}(\xi(x), \eta(x))=\frac{1}{m !} \sum_{l+r=m} \partial_{1}^{l} \partial_{2}^{r} g(0,0) \xi(x)^{l} \eta(x)^{r},
$$

and this gives the statement, since $g_{m}$ satisfies the same properties as $g$.

The fact that $P$ belongs to the class $\mathcal{T}^{\nu, \beta}$ is directly related to the distribution of the $\phi_{j}$ 's. Actually we have 
Proposition 3.3. - Let $\nu>1 / 8$ and $0 \leq \beta \leq \frac{1}{24}$. For each $k \geq 1$ and for each $N \geq 0$ there exists $c_{N}>0$ such that for all $j \in \overline{\mathbb{N}}^{k}$

$$
\left|\int_{\mathbb{R}} \phi_{j_{1}} \ldots \phi_{j_{k}} d x\right| \leq c_{N} \frac{\mu(j)^{\nu}}{C(j)^{\beta}} A(j)^{N} \text {. }
$$

As a consequence, any $P$ of the general form (3.4) is in the class $\mathcal{T}^{\nu}$.

The proof will be done in the multidimensional case in the next section (cf. Proposition [3.6).

We can now apply our Theorem 2.23 to obtain

Theorem 3.4. - Assume that $M \in F_{m}$ defined in Proposition 3.1 and that $g \in C^{\infty}\left(\mathbb{C}^{2}, \mathbb{C}\right)$ is real i.e. $g(z, \bar{z}) \in \mathbb{R}$ and has a zero of order at least 3 at the origin. For any $r \geq 3$ there exists $s_{0}(r)$ an integer such that for any $s \geq s_{0}(r)$, there exist $\varepsilon_{0}>0$ and $C>0$ such that if $\left\|\psi_{0}\right\|_{\tilde{H}^{2 s}}=\varepsilon<\varepsilon_{0}$ the equation

$$
i \psi_{t}=\left(-\Delta+x^{2}+M\right) \psi+\partial_{2} g(\psi, \bar{\psi}), \quad x \in \mathbb{R}, t \in \mathbb{R}
$$

with Cauchy data $\psi_{0}$ has a unique solution $\psi \in C^{1}\left(\left(-T_{\epsilon}, T_{\epsilon}\right), \tilde{H}^{2 s}\right)$ with

$$
T_{\epsilon} \geq C \epsilon^{-r} \text {. }
$$

Moreover

(i) $\|\psi(t, \cdot)\|_{\tilde{H}^{2 s}} \leq 2 \epsilon$ for any $t \in\left(-T_{\epsilon}, T_{\epsilon}\right)$.

(ii) $\left.\sum_{j \geq 1} j^{2 s}|| \xi_{j}(t)\right|^{2}-\left|\xi_{j}(0)\right|^{2} \mid \leq \varepsilon^{3}$ for any $t \in\left(-T_{\epsilon}, T_{\epsilon}\right)$ where $\left|\xi_{j}(t)\right|^{2}, j \geq 1$ are the actions of $\psi(t, \cdot)=\sum \xi_{j}(t) \phi_{j}$.

(iii) there exists a torus $\mathcal{T}_{0} \subset \tilde{H}^{2 s}$ such that,

$$
\operatorname{dist}_{2 s}\left(\psi(t, \cdot), \mathcal{T}_{0}\right) \leq C \varepsilon^{r_{1} / 2} \quad \text { for }|t| \leq \epsilon^{-r_{2}}
$$

where $r_{1}+r_{2}=r+3$ and dist $t_{2 s}$ denotes the distance on $\tilde{H}^{2 s}$ associated with the norm $\|\cdot\|_{\tilde{H}^{2 s}}$.

Proof. - Let $\psi_{0}=\sum \xi_{j}(0) \phi_{j}$ and denote $z_{0}=(\xi(0), \bar{\xi}(0))$. Notice that if $\psi_{0} \in \tilde{H}^{2 s}$ with $\left\|\psi_{0}\right\|_{\tilde{H}^{2 s}}=\epsilon$ then $z_{0} \in \mathcal{P}_{s}$ and $\left\|z_{0}\right\|_{s}=\epsilon$. Denote by $z(t)$ the solution of the Cauchy problem $\dot{z}=X_{H}(z), z(0)=z_{0}$, where $H=H_{0}+P$ is the Hamiltonian function associated to the equation (3.7) written in the Hermite decomposition $\psi(t)=\sum \xi_{j}(t) \phi_{j}, z(t)=(\xi(t), \bar{\xi}(t))$. We note that, since $P$ is real, $z$ remains a real point of $\mathcal{P}_{s}$ for all $t$ and that $\|\psi(t)\|_{\tilde{H}^{2 s}}=\|z(t)\| s$.

Then we denote by $z^{\prime}=\tau^{-1}(z)$ where $\tau: \mathcal{V}_{s} \rightarrow \mathcal{U}_{s}$ is the transformation given by Theorem 2.23 (so that $z^{\prime}$ denotes the normalized coordinates) associated to the order $r+2$ and $s \geq s_{0}(r+2)$ given by the same Theorem. We note that, since the transformation $\tau$ is generated by a real Hamiltonian, $z^{\prime}(t)$ is still a real point. 
Let $\varepsilon_{0}>0$ be such that $B_{2 \varepsilon_{0}} \subset \mathcal{V}_{s}$ and take $0<\varepsilon<\varepsilon_{0}$. We assume that $\|z(0)\|_{s}=\left\|\psi_{0}\right\|_{\tilde{H}^{s}}=\varepsilon$. For $z=(\xi, \eta) \in \mathcal{P}_{s}$ we define

$$
N(z):=2 \sum_{j=1}^{\infty} j^{2 s} I_{j}(\xi, \eta)
$$

where we recall that $I_{j}(\xi, \eta)=\xi_{j} \eta_{j}$. We notice that for a real point $z=$ $(\xi, \bar{\xi}) \in \mathcal{P}_{s}$

$$
N(z)=\|z\|_{s}^{2}
$$

Thus in particular we have

$$
N(z(t))=\|z(t)\|_{s}^{2} \quad \text { and } N\left(z^{\prime}(t)\right)=\left\|z^{\prime}(t)\right\|_{s}^{2} .
$$

Using that $Z$ depends only on the normalized actions, we have

$$
\dot{N}\left(z^{\prime}\right)=\{N, H \circ \tau\} \circ \tau^{-1}(z)=\{N, R\}\left(z^{\prime}\right) .
$$

Therefore as far as $\|z(t)\|_{s} \leq 2 \varepsilon$, and thus $z(t) \in \mathcal{V}_{s}$, by assertion (c) of Theorem 2.23, $\left\|z^{\prime}(t)\right\|_{s} \leq C \varepsilon$ and using (3.9) and assertion (b) of Theorem 2.23 (at order $r+2$ ) we get

$$
\left|N\left(z^{\prime}(t)\right)-N\left(z^{\prime}(0)\right)\right| \leq\left|\int_{0}^{t}\{N, R\}\left(z^{\prime}\left(t^{\prime}\right)\right) d t^{\prime}\right| \leq C t\left\|z^{\prime}(t)\right\|_{s}^{r+3} \leq C t \varepsilon^{r+3} .
$$

In particular, as far as $\|z(t)\|_{s} \leq 2 \varepsilon$ and $|t| \leq C \varepsilon^{-r}$

$$
\left|N\left(z^{\prime}(t)\right)-N\left(z^{\prime}(0)\right)\right| \leq C \varepsilon^{3} .
$$

Therefore using again assertion (c) of Theorem 2.23, we obtain

$$
|N(z(t))-N(z(0))| \leq C \varepsilon^{3}
$$

which, choosing $\varepsilon_{0}$ small enough, leads to $\|z(t)\|_{s} \leq 3 / 2 \varepsilon$ as long as $\|z(t)\|_{s} \leq$ $2 \varepsilon$ and $|t| \leq C \varepsilon^{-r}$. Thus (3.8) and assertions (i) follow by a continuity argument.

To prove assertion (ii) we recall the notation $I_{j}(z)=I_{j}(\xi, \eta)=\xi_{j} \eta_{j}$ for the actions associated to $z=(\xi, \eta)$. Using that $Z$ depends only on the actions, we have

$$
\left\{I_{j} \circ \tau^{-1}, H\right\}(z)=\left\{I_{j}, H \circ \tau\right\} \circ \tau^{-1}(z)=\left\{I_{j}, R\right\}\left(z^{\prime}\right) .
$$

Therefore, we get in the normalized coordinates

$$
\frac{d}{d t} I_{j}\left(\xi^{\prime}, \eta^{\prime}\right)=-i \xi_{j}^{\prime} \frac{\partial R}{\partial \eta_{j}}+i \eta_{j}^{\prime} \frac{\partial R}{\partial \xi_{j}}
$$

\footnotetext{
${ }^{(5)}$ That is precisely at this point that we need to work with real Hamiltonians. The Birkhoff normal form theorem is essentially algebraic and does hold for complex Hamiltonians.
} 
and thus

$$
\begin{aligned}
\sum_{j} j^{2 s}\left|\frac{d}{d t} I_{j}\left(\xi^{\prime}, \bar{\xi}^{\prime}\right)\right| & =\sum_{j} j^{2 s}\left|-\xi_{j}^{\prime} \frac{\partial R}{\partial \eta_{j}}+\bar{\xi}_{j}^{\prime} \frac{\partial R}{\partial \xi_{j}}\right| \\
& \leq\left(\sum_{j} j^{2 s}\left(\left|\xi_{j}^{\prime}\right|^{2}+\left|\xi_{j}^{\prime}\right|^{2}\right)\right)^{1 / 2}\left(\sum_{j} j^{2 s}\left(\left|\frac{\partial R}{\partial \eta_{j}}\right|^{2}+\left|\frac{\partial R}{\partial \xi_{j}}\right|^{2}\right)\right)^{1 / 2}
\end{aligned}
$$

which leads to

$$
\sum_{j} j^{2 s}\left|\frac{d}{d t} I_{j}\left(z^{\prime}\right)\right| \leq\left\|z^{\prime}\right\|_{s}\left\|X_{R}\left(z^{\prime}\right)\right\|_{s} \leq\left\|z^{\prime}\right\|_{s}^{r+3} .
$$

Thus, recalling that $I_{j}\left(\xi^{\prime}, \bar{\xi}^{\prime}\right)=\left|\xi_{j}^{\prime}\right|^{2}$ we get

$$
\left.\sum_{j \geq 1} j^{2 s}|| \xi_{j}^{\prime}(t)\right|^{2}-\left|\xi_{j}^{\prime}(0)\right|^{2} \mid \leq \varepsilon^{3} \text { for any }|t| \leq C \varepsilon^{-r} .
$$

On the other hand, using (i) and assertion (c) of Theorem 2.23 , for any $|t| \leq$ $C \varepsilon^{-r}$, one has

$$
\left.\left.\sum_{j \geq 1} j^{2 s}|| \xi_{j}(t)\right|^{2}\right)-\left|\xi_{j}^{\prime}(t)\right|^{2}\left|\leq \sum_{j \geq 1} j^{2 s}\left(\left|\xi_{j}(t)\right|+\left|\xi_{j}^{\prime}(t)\right|\right)\right| \xi_{j}(t)-\xi_{j}^{\prime}(t) \mid \leq C \varepsilon^{3} .
$$

Combining this last relation with (3.11), assertion (ii) follows.

To prove (iii), let $\bar{I}_{j}=I_{j}^{\prime}(0)$ be the initial actions in the normalized coordinates and define the smooth torus

$$
\Pi_{0}:=\left\{z \in \mathcal{P}_{s}: I_{j}(z)=\bar{I}_{j}, j \geq 1\right\}
$$

and its image in $\tilde{H}^{s}$

$$
\mathcal{T}_{0}=\left\{u \in \tilde{H}^{s}: u=\sum \xi_{j} \phi_{j} \text { with } \tau(\xi, \bar{\xi}) \in \Pi_{0}\right\} .
$$

We have

$$
d_{s}\left(z(t), \mathcal{T}_{0}\right) \leq\left[\sum_{j} j^{2 s}\left|\sqrt{I_{j}^{\prime}(t)}-\sqrt{\bar{I}_{j}}\right|^{2}\right]^{1 / 2}
$$

where $d_{s}$ denotes the distance in $\mathcal{P}_{s}$ associated to $\|\cdot\|_{s}$. Notice that for $a, b \geq 0$,

$$
|\sqrt{a}-\sqrt{b}| \leq \sqrt{|a-b|}
$$


Thus, using (3.10), we get

$$
\begin{aligned}
{\left[d_{s}\left(z(t), \mathcal{T}_{0}\right)\right]^{2} } & \leq \sum_{j} j^{2 s}\left|I_{j}^{\prime}(t)-I_{j}^{\prime}(0)\right| \\
& \leq|t| \sum_{j} j^{2 s}\left|\dot{I}_{j}^{\prime}(t)\right| \\
& \leq \frac{1}{\epsilon^{r_{1}}}\left\|z^{\prime}\right\|_{s}\left\|X_{R}\left(z^{\prime}\right)\right\|_{s} \\
& \leq C \frac{1}{\epsilon^{r_{1}}} \epsilon^{r+3} \leq C \epsilon^{r+3-r_{1}} .
\end{aligned}
$$

which gives (ii).

\subsection{Multidimensional nonlinear harmonic oscillator. -}

3.2.1. Model. - The spectrum of the d-dimensional harmonic oscillator

$$
T=-\Delta+|x|^{2}=-\Delta+x_{1}^{2}+\cdots+x_{d}^{2}
$$

is the sum of $d$-copies of the odd integers set, i.e. the spectrum of $T$ equals $\mathbb{N}_{d}$ with

$$
\mathbb{N}_{d}=\left\{\begin{array}{c}
2 \mathbb{N} \backslash\{0,2, \cdots, d-2\} \text { if } d \text { is even } \\
2 \mathbb{N}+1 \backslash\{1,3, \cdots, d-2\} \text { if } d \text { is odd }
\end{array}\right.
$$

For $j \in \mathbb{N}_{d}$ we denote the associated eigenspace $E_{j}$ which dimension is

$$
d_{j}=\sharp\left\{\left(i_{1}, \cdots, i_{d}\right) \in(2 \mathbb{N}+1)^{d} \mid i_{1}+\cdots+i_{d}=j\right\} .
$$

We denote $\left\{\Phi_{j, l}, l=1, \cdots, d_{j}\right\}$, the basis of $E_{j}$ obtained by $d$-tensor product of Hermite functions: $\Phi_{j, l}=\phi_{i_{1}} \otimes \cdots \phi_{i_{d}}$ with $i_{1}+\cdots+i_{d}=j$.

The Hermite multiplier $M$ is defined on the basis $\left(\Phi_{j, l}\right)_{j \in \mathbb{N}_{d}, l=1, \cdots, d_{j}}$ of $L^{2}\left(\mathbb{R}^{d}\right)$ by

$$
M \Phi_{j, l}=m_{j, l} \Phi_{j, l}
$$

where $\left(m_{j, l}\right)_{j \in \mathbb{N}_{d}, l=1, \cdots, d_{j}}$ is a bounded sequence of real numbers.

The linear part of (1.1) reads

$$
H_{0}=-\Delta+x^{2}+M
$$

$H_{0}$ is still diagonalized by $\left(\Phi_{j, l}\right)_{j \in \mathbb{N}_{d}, l=1, \cdots, d_{j}}$ and the spectrum of $H_{0}$ is

$$
\sigma\left(H_{0}\right)=\left\{j+m_{j, l} \mid j \in \mathbb{N}_{d}, l=1, \cdots, d_{j}\right\}
$$

For simplicity, we will focus on the case $m_{j, l}=m_{j}$ for all $l=1, \cdots, d_{j}$. In this case we have $\sigma\left(H_{0}\right)=\left\{j+m_{j} \mid j \in \mathbb{N}_{d}\right\}$ and, as a consequence of Proposition 3.1 , 
Proposition 3.5. - There exists a set $F_{k} \subset \mathcal{W}_{k}$ whose measure equals 1 such that if $m=\left(m_{j}\right)_{j \in \overline{\mathbb{N}}} \in F_{k}$ then the frequency vector $\left(\omega_{j, i}\right)_{j \in \mathbb{N}_{d}, i=1, \cdots, d_{j}}$ satisfies the following:

for any $r \in \overline{\mathbb{N}}$, there are $\gamma>0$ and $\delta>0$ such that for any $j \in \mathbb{N}_{d}{ }^{r}$, any $l \in\left\{1, \cdots, d_{j_{1}}\right\} \times \cdots \times\left\{1, \cdots, d_{j_{r}}\right\}$ and any $1 \leq i \leq r$, one has

$$
\left|\omega_{j_{1}, l_{1}}+\cdots+\omega_{j_{i}, l_{i}}-\omega_{j_{i+1}, l_{i+1}}-\cdots-\omega_{j_{r}, l_{r}}\right| \geq \gamma \frac{1+S(j)}{\mu(j)^{\delta}}
$$

except if $\left\{j_{1}, \ldots, j_{i}\right\}=\left\{j_{i+1}, \ldots, j_{r}\right\}$.

Concerning the product of eigenfunctions we have,

Proposition 3.6. - Let $\nu>d / 8$. For any $k \geq 1$ and any $N \geq 1$ there exists $c_{N}>0$ such that for any $j \in \mathbb{N}_{d}^{k}$, any $l \in\left\{1, \cdots, d_{j_{1}}\right\} \times \cdots \times\left\{1, \cdots, d_{j_{k}}\right\}$

$$
\left|\int_{\mathbb{R}^{d}} \Phi_{j_{1}, l_{1}} \ldots \Phi_{j_{k}, l_{k}} d x\right| \leq c_{N} \frac{\mu(j)^{\nu}}{C(j)^{\frac{1}{24}}} A(j)^{N} .
$$

Notice that this condition does not distinguish between modes having the same energy.

Proof. - We use the approach developed in [Bam07] Section 6.2. The basic idea lies in the following commutator lemma: Let $A$ be a linear operator which maps $D\left(T^{k}\right)$ into itself and define the sequence of operators

$$
A_{N}:=\left[T, A_{N-1}\right], \quad A_{0}:=A
$$

then ([Bam07 Lemma 7) for any $j_{1} \neq j_{2}$ in $\mathbb{N}_{d}$, any $0 \leq l_{1} \leq d_{1}, 0 \leq l_{2} \leq d_{2}$ and any $N \geq 0$

$$
\left|\left\langle A \Phi_{j_{2}, l_{2}}, \Phi_{j_{1}, l_{1}}\right\rangle\right| \leq \frac{1}{\left|j_{1}-j_{2}\right|^{N}}\left|\left\langle A_{N} \Phi_{j_{2}, l_{2}}, \Phi_{j_{1}, l_{1}}\right\rangle\right| .
$$

Let $A$ be the operator given by the multiplication by the function $\Phi=$ $\Phi_{j_{3}, l_{3}} \cdots \Phi_{j_{k}, l_{k}}$ then by an induction argument

$$
A_{N}=\sum_{0 \leq|\alpha| \leq N} C_{\alpha, N} D^{\alpha}
$$

where

$$
C_{\alpha, N}=\sum_{0 \leq|\beta| \leq 2 N-|\alpha|} V_{\alpha, \beta, N}(x) D^{\beta} \phi
$$


and $V_{\alpha, \beta, N}$ are polynomials of degree less than $2 N$. Therefore one gets

$$
\begin{aligned}
\left|\int_{\mathbb{R}^{d}} \Phi_{j_{1}, l_{1}} \ldots \Phi_{j_{k}, l_{k}} d x\right| & \leq \frac{1}{\left|j_{1}-j_{2}\right|^{N}}\left\|A_{N} \Phi_{j_{2}, l_{2}}\right\|_{L^{2}} \\
& \leq C \frac{1}{\left|j_{1}-j_{2}\right|^{N}} \sum_{0 \leq|\alpha| \leq N} \sum_{0 \leq|\beta| \leq 2 N-|\alpha|}\left\|V_{\alpha, \beta, N} D^{\beta} \phi D^{\alpha} \Phi_{j_{2}, l_{2}}\right\|_{L^{2}} \\
& \leq C \frac{1}{\left|j_{1}-j_{2}\right|^{N}} \sum_{0 \leq|\alpha| \leq N} \sum_{0 \leq|\beta| \leq 2 N-|\alpha|}\left\|\Phi_{j_{2}, l_{2}}\right\|_{|\alpha|}\|\Phi\|_{\nu_{0}+|\beta|}
\end{aligned}
$$

where we used in the last estimate (in this proof, $\|f\|_{s}=\|f\|_{H^{s}\left(\mathbb{R}^{d}\right)}$, the standard Sobolev norm)

$$
\forall \nu_{0}>d / 2 \quad\|f g\|_{L^{2}} \leq C_{\nu_{0}}\|f\|_{\nu_{0}}\|g\|_{L^{2}}
$$

We now estimate $\|\Phi\|_{\nu_{0}+|\beta|}$. First notice that, since $T \Phi_{j, l}=j \Phi_{j, l}$, one has for all $s \geq 0$

$$
\left\|\Phi_{j, l}\right\|_{s} \leq C j^{s / 2}
$$

Then we recall that the Hermite eigenfunctions are uniformly bounded, and in fact (see [Sze75] or [KT05])

$$
\left\|\phi_{j}\right\|_{L^{\infty}} \leq C j^{-1 / 12}
$$

and thus, since $\Phi_{j, l}=\phi_{i_{1}} \otimes \cdots \phi_{i_{d}}$ with $i_{1}+\cdots+i_{d}=j$, we deduce

$$
\left\|\Phi_{j, l}\right\|_{L^{\infty}} \leq C_{d} j^{-1 / 12}
$$

with $C_{d}=C d^{1 / 12}$. Thus using tame estimates (see for instance Tay91)

$$
\|u v\|_{s} \leq C\left(\|u\|_{s}\|v\|_{L^{\infty}}+\|v\|_{s}\|u\|_{L^{\infty}}\right)
$$

combined with (3.19) and (3.21), we get for $j_{3} \geq \cdots \geq j_{k}$,

$$
\|\Phi\|_{s} \leq C j_{3}^{s / 2}
$$


Inserting (3.19) and (3.22) in (3.18) we get

$$
\begin{aligned}
\left|\int_{\mathbb{R}^{d}} \Phi_{j_{1}, l_{1}} \ldots \Phi_{j_{k}, l_{k}} d x\right| & \leq C \frac{1}{\left|j_{1}-j_{2}\right|^{N}} \sum_{0 \leq|\alpha| \leq N} \sum_{0 \leq|\beta| \leq 2 N-|\alpha|} j_{2}^{|\alpha| / 2} j_{3}^{\left.\left(\nu_{0}+|\beta|\right) / 2\right)} \\
& \leq C \frac{1}{\left|j_{1}-j_{2}\right|^{N}} \sum_{0 \leq|\alpha| \leq N} j_{2}^{|\alpha| / 2} j_{3}^{\nu_{0} / 2+N-|\alpha| / 2} \\
& \leq C \frac{1}{\left|j_{1}-j_{2}\right|^{N}} j_{3}^{N+\nu_{0} / 2}\left(\frac{j_{2}}{j_{3}}\right)^{N / 2} \\
& =C \frac{1}{\left|j_{1}-j_{2}\right|^{N}} j_{3}^{\nu_{0} / 2}\left(j_{2} j_{3}\right)^{N / 2} .
\end{aligned}
$$

Now remark that if $\sqrt{j_{2} j_{3}} \leq\left|j_{1}-j_{2}\right|$ then the last estimate implies

$$
\left|\int_{\mathbb{R}^{d}} \Phi_{j_{1}, l_{1}} \ldots \Phi_{j_{k}, l_{k}} d x\right| \leq C j_{3}^{\nu_{0} / 2} \frac{\left(j_{2} j_{3}\right)^{N / 2}}{\left(\sqrt{j_{2} j_{3}}+\left|j_{1}-j_{2}\right|\right)^{N}}=C \mu(j)^{\nu / 2} A(j)^{N}
$$

while if $\sqrt{j_{2} j_{3}}>\left|j_{1}-j_{2}\right|$ then $A(j) \geq 1 / 2$ and thus (3.23) is trivially true.

On the other hand, using (3.21) one has

$$
\left|\int_{\mathbb{R}^{d}} \Phi_{j_{1}, l_{1}} \ldots \Phi_{j_{k}, l_{k}} d x\right| \leq C j_{1}^{-1 / 12}=C C(j)^{-1 / 12} .
$$

Combining this estimate with (3.23) one gets for all $N \geq 1$

$$
\left|\int_{\mathbb{R}^{d}} \Phi_{j_{1}, l_{1}} \ldots \Phi_{j_{k}, l_{k}} d x\right| \leq c_{N} \frac{\mu(j)^{\nu}}{C(j)^{\frac{1}{24}}} A(j)^{N}
$$

with $\nu=\frac{\nu_{0}}{4}$.

3.2.2. Result. - We first generalize the normal form theorem to a context adapted to the multidimensional case. We follow the presentation of Section 2 and only focus on the new features.

Let $s \geq 0$, we consider the phase space $\mathcal{Q}_{s}=\mathcal{L}_{s} \times \mathcal{L}_{s}$ with

$$
\mathcal{L}_{s}=\left\{\left.\left(a_{j, l}\right)_{j \in \mathbb{N}_{d}, 1 \leq l \leq d_{j}}\left|\sum_{j \in \mathbb{N}_{d}}\right| j\right|^{2 s} \sum_{l=1}^{d_{j}}\left|a_{j, l}\right|^{2}<\infty\right\}
$$

that we endow with the standard norm and the standard symplectic structure as for $\mathcal{P}_{s}$ in Section 2.1. Writing $\psi=\sum \xi_{j, l} \Phi_{j, l}, \bar{\psi}=\sum \eta_{j, l} \Phi_{j, l}$ with $(\xi, \eta) \in$ $\mathcal{Q}_{s}$, we note that $\psi \in \tilde{H}^{2 s}$ if and only if $\xi \in \mathcal{L}_{s}$. The linear part of the 
multidimensional version of the linear part of (1.1) reads

$$
H_{0}(\xi, \eta)=\frac{1}{2} \sum_{j \in \mathbb{N}_{d}} \sum_{l=1}^{d_{j}} \omega_{j, l} \xi_{j, l} \eta_{j, l}
$$

For $j \geq 1$, we define

$$
J_{j}(\xi, \eta)=\sum_{l=1}^{d_{j}} \xi_{j, l} \eta_{j, l} .
$$

Using notations of Section 2.1, we define the class $\mathbb{T}_{k}^{\nu}$ of homogeneous polynomials of degree $k$ on $\mathcal{Q}_{s}$

$$
Q(\xi, \eta) \equiv Q(z)=\sum_{j \in \mathbb{N}_{d} k} \sum_{l_{1}=1}^{d_{j_{1}}} \ldots \sum_{l_{m}=1}^{d_{j_{k}}} a_{j, l} z_{j_{1}, l_{1}} \ldots z_{j_{k}, l_{k}}
$$

such that for each $N \geq 1$, there exists a constant $C>0$ such that for all $j, l$

$$
\left|a_{j, l}\right| \leq C \frac{\mu(j)^{\nu}}{C(j)^{1 / 24}} A(j)^{N} .
$$

Then, following Definition 2.11 we define a corresponding class $\mathbb{T}^{\nu}$ of $C^{\infty}$ Hamiltonians on $\mathcal{Q}_{s}$ having their Taylor polynomials in $\mathbb{T}_{k}^{\nu}$. Similarly, following Definition 2.1, we also define $\mathcal{H}_{d}^{s}$ the class of real Hamiltonians $P$ satisfying $P, P_{k} \in C^{\infty}\left(\mathcal{U}_{s}, \mathbb{C}\right)$ and $X_{P}, X_{P_{k}} \in C^{\infty}\left(\mathcal{U}_{s}, \mathcal{Q}_{s}\right)$ for some $\mathcal{U}_{s} \subset \mathcal{Q}_{s}$ a neighborhood of the origin and for all $k \geq 1$ (as before $P_{k}$ denotes the Taylor polynomial of $P$ of degree $k$ ).

In equation (1.1), the Hamiltonian perturbation reads

$$
P(\xi, \eta)=\int_{\mathbb{R}^{d}} g(\xi(x), \eta(x)) d x
$$

where $g$ is $C^{\infty}$ on a neighborhood of 0 in $\mathbb{C}^{2}, \xi(x)=\sum_{j \geq 1} \xi_{j} \phi_{j}(x), \eta(x)=$ $\sum_{j \geq 1} \eta_{j} \phi_{j}(x)$ and $\left(\left(\xi_{j}\right)_{j \geq 1},\left(\eta_{j}\right)_{j \geq 1}\right) \in \mathcal{P}_{s}$. As in the one dimensional case (cf. Lemma 3.2),$P$ belongs to $\mathcal{H}_{d}^{s}$ for $s$ large enough $(s>d / 2)$ and using Proposition 3.6, $P$ belongs to the class $\mathbb{T}^{\nu}$. Therefore one has

Lemma 3.7. - Let $P$ given by (3.24) with $g$ smooth, real and having a zero of order at least 3 at the origin. Then $P \in \mathcal{H}^{s} \cap \mathbb{T}^{\nu}$ for all $s>d / 2$ and for $\nu>d / 8$.

We also need a $d$-dimensional definition of normal form homogeneous polynomial : 
Definition 3.8. - Let $k=2 m$ be an even integer, a formal polynomial $Z$ homogeneous of degree $k$ on $\mathcal{Q}_{s}$ is in normal form if it reads

$$
Z(\xi, \eta)=\sum_{j \in \mathbb{N}_{d}^{k}} \sum_{l_{1}, l_{1}^{\prime}=1}^{d_{j_{1}}} \ldots \sum_{l_{k}, l_{k}^{\prime}=1}^{d_{j_{k}}} a_{j, l, l^{\prime}} \xi_{j_{1}, l_{1}} \eta_{j_{1}, l_{1}^{\prime}} \ldots \xi_{j_{k}, l_{k}} \eta_{j_{k}, l_{k}^{\prime}}
$$

for all $(\xi, \eta) \in \mathcal{Q}_{s}$.

One easily verifies that if $Z$ is in normal form then $Z$ commutes with each $J_{j}=\sum_{l=1}^{d_{j}} \xi_{j, l} \eta_{j, l}$ since for instance

$$
\left\{\xi_{j_{1}, l_{1}} \eta_{j_{1}, l_{1}^{\prime}}, \xi_{j_{1}, l_{1}} \eta_{j_{1}, l_{1}}+\xi_{j_{1}, l_{1}^{\prime}} \eta_{j_{1}, l_{1}^{\prime}}\right\}=0
$$

Modifying slightly the proof of Theorem 2.23 we get

Theorem 3.9. - Let $P$ be a real Hamiltonian belonging in $\mathbb{T}^{\nu} \cap \mathcal{H}_{d}^{s}$ for some $\nu \geq 0$ and for all $s$ sufficiently large and let $\omega$ be a weakly non resonant frequency vector in the sense of (3.16). Then for any $r \geq 3$ there exists $s_{0}$ and for any $s \geq s_{0}$ there exists $\mathcal{U}, \mathcal{V}$ neighborhoods of the origin in $\mathcal{Q}_{s}$ and $\tau: \mathcal{V} \rightarrow \mathcal{U}$ a real analytic canonical transformation which puts $H=H_{0}+P$ in normal form up to order $r$ i.e.

$$
H \circ \tau=H_{0}+Z+R
$$

with

(i) $Z$ is a real continuous polynomial of degree $r$ which belongs to $\mathcal{H}_{d}^{s}$ and which is in normal form in the sense of Definition 3.8. In particular $Z$ commutes with all $J_{j}, j \geq 1$, i.e. $\left\{Z, J_{j}\right\}=0$ for all $j \geq 1$.

(ii) $R$ is real and belongs to $\mathcal{H}_{d}^{s}$, furthermore $\left\|X_{R}(z)\right\|_{s} \leq C_{s}\|z\|_{s}^{r}$ for all $z \in \mathcal{V}_{s}$.

(iii) $\tau$ is close to the identity: $\|\tau(z)-z\|_{s} \leq C_{s}\|z\|_{s}^{2}$ for all $z \in \mathcal{V}_{s}$.

Proof. - The only new point when comparing with Theorem 2.23 , is that in assertion (ii) we obtain $\left\{Z, J_{j}\right\}=0$ for all $j \geq 1$ instead of $\left\{Z, I_{j}\right\}=0$ for all $j \geq 1$. Actually, in view of (3.16), we adapt Lemma 2.22, and in particular (2.30) and (2.31), in such a way $\chi \in \mathbb{T}^{\nu,+}$ and $Z$ is in normal form in the sense of Definition 3.8.

On the other hand, we also verify, following the lines of the proof of assertion (iv) of Proposition 2.13, that a homogeneous polynomial of degree $k+1$ in normal form $Z \in \mathbb{T}^{\nu}$ satisfies $\left\|X_{Z}(z)\right\|_{s} \leq C\|z\|_{s}^{k}$ for all $z$ in a neighborhood of the origin. In particular, if $Z \in \mathbb{T}^{\nu}$ is in normal form, it automatically belongs to $\mathcal{H}_{d}^{s}$ (this point was crucial in the proof of Theorem 2.23). 
Notice that the normal form $H_{0}+Z$ is no longer, in general, integrable. The dynamical consequences are the same as in Theorem 3.4 (i) and (ii) but we have to replace $I_{j}$ by $J_{j}$ in the second assertion. Actually the $J_{j}$ play the rôle of almost actions: they are almost conserved quantities.

Theorem 3.10. - Assume that $m \in F_{k}$ defined in proposition 3.5 and that $g$ is $C^{\infty}$ on a neighborhood of 0 in $\mathbb{C}^{2}, g$ is real i.e. $g(z, \bar{z}) \in \mathbb{R}$ and $g$ vanishes at least at order 3 at the origin. For each $r \geq 3$ and $s \geq s_{0}(r)$, there exists $\varepsilon_{0}>0$ and $c>0$ such that for any $\psi_{0}$ in $\tilde{H}^{s}$, any $\epsilon \in\left(0, \epsilon_{0}\right)$, the equation

$$
i \psi_{t}=\left(-\Delta+x^{2}+M\right) \psi+\partial_{2} g(\psi, \bar{\psi}), \quad x \in \mathbb{R}^{d}, t \in \mathbb{R}
$$

with Cauchy data $\psi_{0}$ has a unique solution $\psi \in C^{1}\left(\left(-T_{\epsilon}, T_{\epsilon}\right), \tilde{H}^{s}\right)$ with

$$
T_{\epsilon} \geq c \epsilon^{-r}
$$

Moreover for any $t \in\left(-T_{\epsilon}, T_{\epsilon}\right)$, one has

$$
\|\psi(t, \cdot)\|_{\tilde{H}^{s}} \leq 2 \epsilon
$$

and

$$
\sum_{j \geq 1} j^{s}\left|J_{j}(t)-J_{j}(0)\right| \leq \varepsilon^{3}
$$

where $J_{j}(t)=\sum_{l=1}^{d_{j}}\left|\xi_{j, l}\right|^{2}, j \geq 1$ are the "pseudo-actions" of $\psi(t, \cdot)=$ $\sum_{j, l} \xi_{j, l}(t) \Phi_{j, l}(\cdot)$.

Proof. - Just remark that as in the proof of Theorem 3.4, defining $N(z):=$ $2 \sum_{j \in \mathbb{N}_{d}} j^{s} J_{j}(\xi, \eta)=2 \sum_{j \in \mathbb{N}_{d}} j^{s} \sum_{l=1}^{d_{j}} \xi_{j, l} \eta_{j, l}$ one has $N(z)=\|z\|_{s / 2}^{2}$ for all real point $z=(\xi, \bar{\xi})$. On the other hand, using that $Z$ commutes with $J_{j}$, we have

$$
\left\{N \circ \tau^{-1}, H\right\}(z)=\{N, H \circ \tau\} \circ \tau^{-1}(z)=\{N, R\}\left(z^{\prime}\right) .
$$

Therefore, in the normalized variables, we have the estimate $|\dot{N}| \leq C N^{(r+1) / 2}$ and the theorem follows as in the proof of Theorem 3.4.

\section{References}

[Bam03] D. Bambusi, Birkhoff normal form for some nonlinear PDEs, Comm. Math. Physics 234 (2003), 253-283.

[Bam07] _ A birkhoff normal form theorem for some semilinear pdes, Hamiltonian Dynamical Systems and Applications, Springer, 2007, pp. 213-247.

[BDGS07] D. Bambusi, J.-M. Delort, B. Grébert, and J. Szeftel, Almost global existence for Hamiltonian semilinear Klein-Gordon equations with small Cauchy data on Zoll manifolds, Comm. Pure Appl. Math. 60 (2007), no. 11, 1665-1690. 
[BG06] D. Bambusi and B. Grébert, Birkhoff normal form for PDEs with tame modulus, Duke Math. J. 135 (2006), 507-567.

[Bou96] J. Bourgain, Construction of approximative and almost-periodic solutions of perturbed linear Schrödinger and wave equations, Geometric and Functional Analysis 6 (1996), 201-230.

[Bou05] J. Bourgain, Green's function estimates for lattice Schrödinger operators and applications, Annals of Mathematics Studies, vol. 158, Princeton University Press, Princeton, NJ, 2005.

[Car02] R. Carles, Remarks on nonlinear Schrödinger equations with harmonic potential, Ann. Henri Poincaré 3 (2002), no. 4, 757-772.

[Cra00] W. Craig, Problèmes de petits diviseurs dans les équations aux dérivées partielles, Panoramas et Synthéses, no. 9, Société Mathématique de France, 2000.

[DS04] J. M. Delort and J. Szeftel, Long-time existence for small data nonlinear Klein-Gordon equations on tori and spheres, Internat. Math. Res. Notices 37 (2004), 1897-1966.

[Gré07] Benoît Grébert, Birkhoff normal form and Hamiltonian PDEs, Partial differential equations and applications, Sémin. Congr., vol. 15, Soc. Math. France, Paris, 2007, pp. 1-46.

[Hel84] Bernard Helffer, Théorie spectrale pour des opérateurs globalement elliptiques, Astérisque, vol. 112, Société Mathématique de France, Paris, 1984, With an English summary.

[KP03] T. Kappeler and J. Pöschel, KAM \& KdV, Springer, 2003.

[KT05] Herbert Koch and Daniel Tataru, $L^{p}$ eigenfunction bounds for the Hermite operator, Duke Math. J. 128 (2005), no. 2, 369-392.

[Kuk87] S. B. Kuksin, Hamiltonian perturbations of infinite-dimensional linear systems with an imaginary spectrum, Funct. Anal. Appl., 21 (1987), 192-205.

[Kuk93] — Nearly integrable infinite-dimensional Hamiltonian systems, Springer-Verlag, Berlin, 1993.

[Kuk00] — Analysis of Hamiltonian PDEs, Oxford University Press, Oxford, 2000.

[PS03] Lev Pitaevskii and Sandro Stringari, Bose-Einstein condensation, International Series of Monographs on Physics, vol. 116, The Clarendon Press Oxford University Press, Oxford, 2003.

[Pös96] J. Pöschel, A KAM-theorem for some nonlinear PDEs, Ann. Scuola Norm. Sup. Pisa, Cl. Sci., IV Ser. 1523 (1996), 119-148.

[Sze75] Gábor Szegő, Orthogonal polynomials, fourth ed., American Mathematical Society, Providence, R.I., 1975, American Mathematical Society, Colloquium Publications, Vol. XXIII.

[Tay91] Michael E. Taylor, Pseudodifferential operators and nonlinear PDE, Progress in Mathematics, vol. 100, Birkhäuser Boston Inc., Boston, MA, 1991.

[Wan08] W.-M. Wang, Pure point spectrum of the Floquet Hamiltonian for the quantum harmonic oscillator under time quasi-periodic perturbations, Comm. Math. Physics 277 (2008), 459-496. 
[YZ04] K. Yajima and G. Zhang, Local smoothing property and Strichartz inequality for Schrödinger equations with potentials superquadratic at infinity, J. Differential Equations 202 (2004), no. 1, 81-110.

[Zha05] Jian Zhang, Sharp threshold for blowup and global existence in nonlinear Schrödinger equations under a harmonic potential, Comm. Partial Differential Equations 30 (2005), no. 10-12, 1429-1443.

\section{Benoît Grébert, Rafik Imekraz, Éric Paturel}

Laboratoire de Mathématiques Jean Leray UMR 6629,

Université de Nantes,

2, rue de la Houssinière,

44322 Nantes Cedex 3, France

benoit.grebert@univ-nantes.fr

E-mail: rafik.imekraz@univ-nantes.fr

eric.paturel@univ-nantes.fr

October 26, 2018

Benoît Grébert, Rafik Imekraz, Eric Paturel 